\title{
ON A HYBRID FINITE-VOLUME-PARTICLE METHOD
}

\author{
Alina Chertock ${ }^{1}$ And Alexander Kurganov ${ }^{2}$
}

\begin{abstract}
We present a hybrid finite-volume-particle numerical method for computing the transport of a passive pollutant by a flow. The flow is modeled by the one- and two-dimensional Saint-Venant system of shallow water equations and the pollutant propagation is described by a transport equation. This paper is an extension of our previous work [Chertock, Kurganov and Petrova, J. Sci. Comput. (to appear)], where the one-dimensional finite-volume-particle method has been proposed. The core idea behind the finite-volume-particle method is to use different schemes for the flow and pollution computations: the shallow water equations are numerically integrated using a finite-volume scheme, while the transport equation is solved by a particle method. This way the specific advantages of each scheme are utilized at the right place. A special attention is given to the recovery of the point values of the numerical solution from its particle distribution. The reconstruction is obtained using a dual equation for the pollutant concentration. This results in a significantly enhanced resolution of the computed solution and also makes it much easier to extend the finite-volume-particle method to the two-dimensional case.
\end{abstract}

Mathematics Subject Classification. 34A36, 35L67, 35Q35, 65M99.

Received: April 19, 2004

\section{INTRODUCTION}

Prediction of pollution transport by flows is an important problem in many industrial and environmental projects. Different mathematical models are used to describe the propagation of the pollutant and to obtain its accurate location and concentration.

In this paper, we consider the transport of a passive pollutant by a flow modeled by the Saint-Venant system of shallow water equations. In the two-dimensional (2-D) case, the system reads

$$
\left\{\begin{array}{l}
h_{t}+(h u)_{x}+(h v)_{y}=S, \\
(h u)_{t}+\left(h u^{2}+\frac{g h^{2}}{2}\right)_{x}+(h u v)_{y}=-g h B_{x} \\
(h v)_{t}+(h u v)_{x}+\left(h v^{2}+\frac{g h^{2}}{2}\right)_{y}=-g h B_{y} .
\end{array}\right.
$$

Keywords and phrases. Shallow water equations, transport of passive pollutant, finite-volume schemes, particle method.

1 Department of Mathematics, North Carolina State University, Raleigh, NC 27695, USA. e-mail: chertock@math.ncsu.edu

2 Department of Mathematics, Tulane University, New Orleans, LA 70118, USA. e-mail: kurganov@math.tulane.edu 
Here, $h, u$, and $v$ are the depth, the $x$ - and $y$-velocities of the water, respectively, $g$ is the gravity constant, and $S$ is a fluid mass source term. The function $B$ represents the bottom topography $(B \equiv$ Const corresponds to the flat bottom case). System (1.1), introduced in [23], is commonly used to describe flows in rivers and coastal areas. For a detailed description of a more realistic shallow water model, derived from the Navier-Stokes equations, we refer the reader to [12].

The propagation of the pollutant is modeled by the transport equation,

$$
(h T)_{t}+(u h T)_{x}+(v h T)_{y}=T_{S} S
$$

which describes the motion of the pollutant mass concentration $T$, where $T_{S}$ is a given concentration of the pollutant at the fluid mass source (see, e.g., [2,4]). Equations (1.2) and (1.1) are coupled through the source, mass and velocity terms.

Designing an accurate, efficient and reliable numerical method for this model is a challenging task due to the following reasons. Solutions of system (1.1)-(1.2) are typically nonsmooth: they may contain both nonlinear shock and rarefaction waves, and linear discontinuities in the pollution concentration. Moreover, the interaction with a nonflat bottom may result in very complicated wave structures and nontrivial equilibria, which are hard to preserve numerically. In addition, dry states (arising, for example, in dam break problems) need special attention, since (even small) numerical oscillations may lead to nonphysical negative values of the water depth there.

In order to overcome these difficulties, a high-resolution shock-capturing numerical method is required. Such methods for hyperbolic systems of balance laws, and in particular for the Saint-Venant system (1.1), are readily available (to cite a few of them, see e.g. $[1,3,11,14,21]$ ). In this paper, we implement semi-discrete central schemes. These schemes were originally introduced in [18] in the context of hyperbolic systems of conservation laws, and then further improved in [15-17], where the so-called central-upwind schemes have been developed. An application of the central-upwind schemes to the Saint-Venant system can be found in [14].

Godunov-type central-upwind schemes enjoy all the major advantages of Riemann-problem-solver-free central schemes (universality, efficiency and robustness), and at the same time, have a certain "built-in" upwind nature. They can be relatively easily extended to solve (1.1)-(1.2), but, as we demonstrate in Section 3, the resolution of the computed contact waves in the pollution concentration is not sufficiently sharp. There are some other alternatives (see e.g. [2]), but we are not aware of any method which completely resolves this issue.

In [5], a hybrid finite-volume-particle method for the one-dimensional (1-D) version of (1.1)-(1.2) has been proposed. The core idea is to use central-upwind schemes to solve the system of balance laws (1.1) and a particle method [22] to solve the transport equation (1.2). The problem, which remained open in [5], is how to avoid the loss of resolution when the point values of the numerical solution are recovered from its particle distribution at the final time. For instance, the standard reconstruction procedure, see e.g. [22], typically smears discontinuities. An alternative reconstruction technique used in [5] does not smear the discontinuities, but may lead to oscillations, which can be filtered out in certain cases. To remove the oscillations, a nonlinear filter from [7] was implemented in [5] as a post-processing. However, the applicability of this filter is limited and its $2-\mathrm{D}$ extension is rather cumbersome.

In this paper, we propose another way of recovering the point values of the computed solution from the particle distribution - the solution is recovered via an equation, dual to (1.2). This prevents the aforementioned loss of resolution and leads to a new finite-volume-particle (FVP) method, which takes an advantage of the low dissipative (in certain cases, completely nondissipative) particle method, and thus guarantees almost perfect resolution of the contact discontinuities in the pollutant concentration. We would like to emphasize that the new recovering procedure not only significantly enhances the resolution of the computed 1-D solutions, but also makes a 2-D extension of the FVP method much easier. Therefore, in this work we present the new 2-D method while leaving the details of its $1-\mathrm{D}$ reduction to the reader (see also [5], where the original 1-D method is described). 
The paper is organized as follows. In Sections 2.1 and 2.2, we give a brief overview of central-upwind schemes and particle methods. Our new method is described in Section 2.3, and the numerical computations are carried out in Section 3.

\section{Hybrid Finite-VOLUME-PARTICLE METHOD}

\subsection{Semi-discrete central-upwind schemes - an overview}

In this section, we briefly describe the central-upwind schemes for 2-D hyperbolic systems of conservation and balance laws. These schemes are Godunov-type finite-volume methods. For their complete description and derivation, as well as for their $1-\mathrm{D}$ version, we refer the reader to $[15,16]$.

We first consider a $2-\mathrm{D}$ system of conservation laws,

$$
u_{t}+f(u)_{x}+g(u)_{y}=0
$$

and note that it can be rewritten in the equivalent form:

$$
\begin{aligned}
\frac{\mathrm{d}}{\mathrm{d} t} \bar{u}(x, y, t)= & -\frac{1}{|I(x, y)|}\left[\int_{\eta=y-\frac{\Delta y}{2}}^{y+\frac{\Delta y}{2}}\left\{f\left(u\left(x+\frac{\Delta x}{2}, \eta, t\right)\right)-f\left(u\left(x-\frac{\Delta x}{2}, \eta, t\right)\right)\right\} \mathrm{d} \eta\right. \\
& \left.+\int_{\xi=x-\frac{\Delta x}{2}}^{x+\frac{\Delta x}{2}}\left\{g\left(u\left(\xi, y+\frac{\Delta y}{2}, t\right)\right)-g\left(u\left(\xi, y-\frac{\Delta y}{2}, t\right)\right)\right\} \mathrm{d} \xi\right]
\end{aligned}
$$

where $\bar{u}(x, y, t):=\frac{1}{|I(x, y)|} \iint_{I(x, y)} u(\xi, \eta, t) \mathrm{d} \eta \mathrm{d} \xi, I(x, y):=\left\{\xi, \eta:|\xi-x|<\frac{\Delta x}{2},|\eta-y|<\frac{\Delta y}{2}\right\}$, and $|I(x, y)|=$ $\Delta x \Delta y$.

For simplicity, we consider a uniform grid $x_{\mu}:=\mu \Delta x, y_{\nu}:=\nu \Delta y$. If at time level $t$ the cell averages, $\bar{u}_{j, k}(t):=\bar{u}\left(x_{j}, y_{k}, t\right)$, are available, we use them to reconstruct a non-oscillatory piecewise polynomial in $x$ and $y$,

$$
\widetilde{u}(x, y, t)=p_{j, k}(x, y, t), \quad x_{j-\frac{1}{2}}<x<x_{j+\frac{1}{2}}, \quad y_{k-\frac{1}{2}}<y<y_{k+\frac{1}{2}}, \quad \forall j, k,
$$

and evolve it according to (2.3). The (formal) order of the resulting scheme depends on the order of accuracy of a quadrature used to approximate the integrals in (2.3) and the order of accuracy of the piecewise polynomial interpolant (2.4). For instance, if second-order accuracy is desired, one may use the midpoint rule for the integrals in $(2.3)$,

$$
\frac{\mathrm{d}}{\mathrm{d} t} \bar{u}_{j, k}(t)=-\frac{f\left(u\left(x_{j+\frac{1}{2}}, y_{k}, t\right)\right)-f\left(u\left(x_{j-\frac{1}{2}}, y_{k}, t\right)\right)}{\Delta x}-\frac{g\left(u\left(x_{j}, y_{k+\frac{1}{2}}, t\right)\right)-g\left(u\left(x_{j}, y_{k-\frac{1}{2}}, t\right)\right)}{\Delta y}
$$

and a piecewise linear reconstruction,

$$
p_{j, k}(x, y, t)=\bar{u}_{j, k}(t)+\left(u_{x}\right)_{j, k}\left(x-x_{j}\right)+\left(u_{y}\right)_{j, k}\left(y-y_{k}\right)
$$

To ensure a non-oscillatory behavior of the second-order central-upwind scheme, the slopes in (2.6) should be computed with a nonlinear limiter. In our numerical experiments, we have used a one-parameter family of the 
minmod limiters [19, 20,24]:

$$
\begin{aligned}
\left(u_{x}\right)_{j, k} & =\operatorname{minmod}\left(\theta \frac{\bar{u}_{j+1, k}-\bar{u}_{j, k}}{\Delta x}, \frac{\bar{u}_{j+1, k}-\bar{u}_{j-1, k}}{2 \Delta x}, \theta \frac{\bar{u}_{j, k}-\bar{u}_{j-1, k}}{\Delta x}\right), \\
\left(u_{y}\right)_{j, k} & =\operatorname{minmod}\left(\theta \frac{\bar{u}_{j, k+1}-\bar{u}_{j, k}}{\Delta y}, \frac{\bar{u}_{j, k+1}-\bar{u}_{j, k-1}}{2 \Delta y}, \theta \frac{\bar{u}_{j, k}-\bar{u}_{j, k-1}}{\Delta y}\right),
\end{aligned}
$$

where $\theta \in[1,2]$, and the multivariate minmod function is defined by

$$
\operatorname{minmod}\left(z_{1}, z_{2}, \ldots\right):= \begin{cases}\min _{j}\left\{z_{j}\right\}, & \text { if } z_{j}>0 \forall j \\ \max _{j}\left\{z_{j}\right\}, & \text { if } z_{j}<0 \quad \text { otherwise } \\ 0, & \text { oth }\end{cases}
$$

Notice that larger $\theta$ 's correspond to less dissipative but, in general, more oscillatory limiters.

The second-order semi-discrete central-upwind scheme,

$$
\frac{\mathrm{d}}{\mathrm{d} t} \bar{u}_{j, k}(t)=-\frac{H_{j+\frac{1}{2}, k}^{x}(t)-H_{j-\frac{1}{2}, k}^{x}(t)}{\Delta x}-\frac{H_{j, k+\frac{1}{2}}^{y}(t)-H_{j, k-\frac{1}{2}}^{y}(t)}{\Delta y}
$$

is, in fact, an approximation of (2.5) with the numerical fluxes $H_{j+\frac{1}{2}, k}^{x}$ and $H_{j, k+\frac{1}{2}}^{y}$ given by (see [15] for the derivation)

$$
H_{j+\frac{1}{2}, k}^{x}(t):=\frac{a_{j+\frac{1}{2}, k}^{+} f\left(u_{j, k}^{\mathrm{E}}\right)-a_{j+\frac{1}{2}, k}^{-} f\left(u_{j+1, k}^{\mathrm{W}}\right)}{a_{j+\frac{1}{2}, k}^{+}-a_{j+\frac{1}{2}, k}^{-}}+a_{j+\frac{1}{2}, k}^{+} a_{j+\frac{1}{2}, k}^{-}\left[\frac{u_{j+1, k}^{\mathrm{W}}-u_{j, k}^{\mathrm{E}}}{a_{j+\frac{1}{2}, k}^{+}-a_{j+\frac{1}{2}, k}^{-}}-q_{j+\frac{1}{2}, k}^{x}\right],
$$

and

$$
H_{j, k+\frac{1}{2}}^{y}(t):=\frac{b_{j, k+\frac{1}{2}}^{+} g\left(u_{j, k}^{\mathrm{N}}\right)-b_{j, k+\frac{1}{2}}^{-} g\left(u_{j, k+1}^{\mathrm{S}}\right)}{b_{j, k+\frac{1}{2}}^{+}-b_{j, k+\frac{1}{2}}^{-}}+b_{j, k+\frac{1}{2}}^{+} b_{j, k+\frac{1}{2}}^{-}\left[\frac{u_{j, k+1}^{\mathrm{S}}-u_{j, k}^{\mathrm{N}}}{b_{j, k+\frac{1}{2}}^{+}-b_{j, k+\frac{1}{2}}^{-}}-q_{j, k+\frac{1}{2}}^{y}\right] .
$$

Here, the one-sided local speeds can be estimated, for example, by

$$
\begin{aligned}
a_{j+\frac{1}{2}, k}^{+} & :=\max \left\{\lambda_{N}\left(A\left(u_{j+1, k}^{\mathrm{W}}\right)\right), \lambda_{N}\left(A\left(u_{j, k}^{\mathrm{E}}\right)\right), 0\right\}, \\
a_{j+\frac{1}{2}, k}^{-} & :=\min \left\{\lambda_{1}\left(A\left(u_{j+1, k}^{\mathrm{W}}\right)\right), \lambda_{1}\left(A\left(u_{j, k}^{\mathrm{E}}\right)\right), 0\right\}, \\
b_{j, k+\frac{1}{2}}^{+} & :=\max \left\{\lambda_{N}\left(B\left(u_{j, k+1}^{\mathrm{S}}\right)\right), \lambda_{N}\left(B\left(u_{j, k}^{\mathrm{N}}\right)\right), 0\right\}, \\
b_{j, k+\frac{1}{2}}^{-} & :=\min \left\{\lambda_{1}\left(B\left(u_{j, k+1}^{\mathrm{S}}\right)\right), \lambda_{1}\left(B\left(u_{j, k}^{\mathrm{N}}\right)\right), 0\right\},
\end{aligned}
$$

where, $\lambda_{1}<\lambda_{2}<\ldots<\lambda_{N}$ are the $N$ eigenvalues of the corresponding Jacobians, $A:=\frac{\partial f}{\partial u}$ and $B:=\frac{\partial g}{\partial u}$, and the point values of the piecewise linear reconstruction (2.6) are given by

$$
u_{j, k}^{\mathrm{E}(\mathrm{W})}:=\bar{u}_{j, k}(t) \pm \frac{\Delta x}{2}\left(u_{x}\right)_{j, k}, \quad u_{j, k}^{\mathrm{N}(\mathrm{S})}:=\bar{u}_{j, k}(t) \pm \frac{\Delta y}{2}\left(u_{y}\right)_{j, k} .
$$


Finally, $q_{j+\frac{1}{2}, k}^{x}$ and $q_{j, k+\frac{1}{2}}^{y}$ are the "anti-diffusion" terms that help to reduce numerical dissipation present at non-oscillatory central schemes [15]:

$$
\begin{aligned}
& q_{j+\frac{1}{2}, k}^{x}=\operatorname{minmod}\left(\frac{u_{j+1, k}^{\mathrm{NW}}-u_{j+\frac{1}{2}, k}^{\mathrm{int}}}{a_{j+\frac{1}{2}, k}^{+}-a_{j+\frac{1}{2}, k}^{-}}, \frac{u_{j+\frac{1}{2}, k}^{\mathrm{int}}-u_{j, k}^{\mathrm{NE}}}{a_{j+\frac{1}{2}, k}^{+}-a_{j+\frac{1}{2}, k}^{-}}, \frac{u_{j+1, k}^{\mathrm{SW}}-u_{j+\frac{1}{2}, k}^{\mathrm{int}}}{a_{j+\frac{1}{2}, k}^{+}-a_{j+\frac{1}{2}, k}^{-}}, \frac{u_{j+\frac{1}{2}, k}^{\mathrm{int}}-u_{j, k}^{\mathrm{SE}}}{a_{j+\frac{1}{2}, k}^{+}-a_{j+\frac{1}{2}, k}^{-}}\right), \\
& q_{j, k+\frac{1}{2}}^{y}=\operatorname{minmod}\left(\frac{u_{j, k+1}^{\mathrm{SW}}-u_{j, k+\frac{1}{2}}^{\mathrm{int}}}{b_{j, k+\frac{1}{2}}^{+}-b_{j, k+\frac{1}{2}}^{-}}, \frac{u_{j, k+\frac{1}{2}}^{\mathrm{int}}-u_{j, k}^{\mathrm{NW}}}{b_{j, k+\frac{1}{2}}^{+}-b_{j, k+\frac{1}{2}}^{-}}, \frac{u_{j, k+1}^{\mathrm{SE}}-u_{j, k+\frac{1}{2}}^{\mathrm{int}}}{b_{j, k+\frac{1}{2}}^{+}-b_{j, k+\frac{1}{2}}^{-}}, \frac{u_{j, k+\frac{1}{2}}^{\mathrm{int}}-u_{j, k}^{\mathrm{NE}}}{b_{j, k+\frac{1}{2}}^{+}-b_{j, k+\frac{1}{2}}^{-}}\right),
\end{aligned}
$$

where

$$
\begin{gathered}
u_{j+\frac{1}{2}, k}^{\mathrm{int}}=\frac{a_{j+\frac{1}{2}, k}^{+} u_{j+1, k}^{\mathrm{W}}-a_{j+\frac{1}{2}, k}^{-} u_{j, k}^{\mathrm{E}}-\left\{f\left(u_{j+1, k}^{\mathrm{W}}\right)-f\left(u_{j, k}^{\mathrm{E}}\right)\right\}}{a_{j+\frac{1}{2}, k}^{+}-a_{j+\frac{1}{2}, k}^{-}}, \\
u_{j, k+\frac{1}{2}}^{\mathrm{int}}=\frac{b_{j, k+\frac{1}{2}}^{+} u_{j, k+1}^{\mathrm{S}}-b_{j, k+\frac{1}{2}}^{-} u_{j, k}^{\mathrm{N}}-\left\{g\left(u_{j, k+1}^{\mathrm{S}}\right)-g\left(u_{j, k}^{\mathrm{N}}\right)\right\}}{b_{j, k+\frac{1}{2}}^{+}-b_{j, k+\frac{1}{2}}^{-}}, \\
u_{j, k}^{\mathrm{NE}(\mathrm{NW})}:=\bar{u}_{j, k}(t) \pm \frac{\Delta x}{2}\left(u_{x}\right)_{j, k}+\frac{\Delta y}{2}\left(u_{y}\right)_{j, k}, \quad u_{j, k}^{\mathrm{SE}(\mathrm{SW})}:=\bar{u}_{j, k}(t) \pm \frac{\Delta x}{2}\left(u_{x}\right)_{j, k}-\frac{\Delta y}{2}\left(u_{y}\right)_{j, k} .
\end{gathered}
$$

Remark. In the original semi-discrete central-upwind scheme [16], both $q_{j+\frac{1}{2}, k}^{x}$ and $q_{j, k+\frac{1}{2}}^{y}$ were chosen to be zero.

In [14], the central-upwind scheme (2.8)-(2.14) has been generalized for the 2-D system of balance laws,

$$
u_{t}+f(u)_{x}+g(u)_{y}=R(u(x, y, t), x, y, t)
$$

The resulting scheme is

$$
\frac{\mathrm{d}}{\mathrm{d} t} \bar{u}_{j, k}(t)=-\frac{H_{j+\frac{1}{2}, k}^{x}(t)-H_{j-\frac{1}{2}, k}^{x}(t)}{\Delta x}-\frac{H_{j, k+\frac{1}{2}}^{y}(t)-H_{j, k-\frac{1}{2}}^{y}(t)}{\Delta y}+\bar{R}_{j, k}(t),
$$

where $\bar{R}_{j, k}(t)$ is an appropriate quadrature for $\frac{1}{\Delta x \Delta y} \int_{x_{j-\frac{1}{2}}}^{x_{j+\frac{1}{2}}} \int_{y_{k-\frac{1}{2}}}^{y_{k+\frac{1}{2}}} R(u(x, y, t), x, y, t) \mathrm{d} x \mathrm{~d} y$, and the numerical fluxes, $H_{j+\frac{1}{2}, k}^{x}, H_{j, k+\frac{1}{2}}^{y}$, are still given by (2.9)-(2.10). However, it should be pointed out that the local speeds, $a_{j+\frac{1}{2}, k}^{ \pm}, b_{j, k+\frac{1}{2}}^{ \pm}$, can be affected by the presence of the source term, and that the formulae in (2.11) may require adjustments.

\section{Remarks.}

1. The semi-discretization (2.9)-(2.15) is a system of time dependent ODEs, which should be solved by at least second-order accurate and stable ODE solver. In our numerical experiments, we have used the third-order strong stability preserving (SSP) Runge-Kutta solver, [13].

2. For the $1-D$ version of the semi-discrete central-upwind scheme $(2.8)-(2.14)$, we refer the reader to $[15-17]$.

\subsection{Particle methods - an overview}

Let us now briefly describe the second main ingredient of our new method - the particle method.

Consider a 2-D linear transport equation with variable coefficients:

$$
\varphi_{t}+\left(\xi_{1} \varphi\right)_{x}+\left(\xi_{2} \varphi\right)_{y}=f(x, y, t)
$$


subject to compactly supported (or periodic) initial data,

$$
\varphi(x, y, 0)=\varphi_{0}(x, y)
$$

and denote by $\mathbf{x}:=(x, y)^{T}$ and $\boldsymbol{\xi}:=\left(\xi_{1}, \xi_{2}\right)^{T}$. The solution of $(2.16)-(2.17)$ is sought as a linear combination of Dirac distributions,

$$
\varphi_{N}(\mathbf{x}, t)=\sum_{i=1}^{N} \alpha_{i}(t) \delta\left(\mathbf{x}-\mathbf{x}_{i}^{p}(t)\right),
$$

where $\mathbf{x}_{i}^{p}$ and $\alpha_{i}$ are the location and the weight of the $i$ th particle, respectively. Considering a weak formulation of the problem and substituting (2.18) into (2.16) results in the following system of ODEs for $\mathbf{x}_{i}^{p}(t)$ and $\alpha_{i}(t)$ :

$$
\left\{\begin{array}{l}
\frac{\mathrm{d} \mathbf{x}_{i}^{p}(t)}{\mathrm{d} t}=\boldsymbol{\xi}\left(\mathbf{x}_{i}^{p}, t\right) \\
\frac{\mathrm{d} \alpha_{i}(t)}{\mathrm{d} t}=\beta_{i}(t)
\end{array}\right.
$$

where $\beta_{i}(t)$ reflects the contribution of the source term $f$ (see, e.g., $[6,22]$ ), which can be approximated by

$$
f(\mathbf{x}, t)=\sum_{i=1}^{N} \beta_{i}(t) \delta\left(\mathbf{x}-\mathbf{x}_{i}^{p}(t)\right), \quad \beta_{i}(t)=\iint_{D_{i}(t)} f(\mathbf{x}, t) \mathrm{d} \mathbf{x} \approx f\left(\mathbf{x}_{i}^{p}(t), t\right) \cdot\left|D_{i}(t)\right| .
$$

Here, $D_{i}(t)$ is the domain that includes the $i$ th particle and satisfies the following properties:

$$
\alpha_{i}(t)=\int_{D_{i}(t)} \int_{(x)} \varphi(\mathbf{x}, t) \mathrm{d} \mathbf{x}, \quad D_{1}(t) \oplus D_{2}(t) \oplus \ldots \oplus D_{N}(t)=\mathbf{X}, \quad \mathbf{X} \text { is the computational domain. }
$$

The size of $D_{i}(t)$ is typically obtained by solving the following ODE:

$$
\frac{\mathrm{d}}{\mathrm{d} t}\left|D_{i}(t)\right|=\left|D_{i}(t)\right| \cdot \operatorname{div} \boldsymbol{\xi}\left(\mathbf{x}_{i}^{p}, t\right)
$$

The initial positions of the particles, $\mathbf{x}_{i}^{p}(0)$, and the weights, $\alpha_{i}(0)$, are chosen to provide with a high-order approximation to the initial data (2.17) according to (2.18). The latter can be achieved by choosing $\alpha_{i}(0)$ to be an integral of $\varphi_{0}(\mathbf{x})$ over $D_{i}(0)$, so that

$$
\sum_{i=1}^{N} \alpha_{i}(0)=\iint_{\mathbf{X}} \varphi_{0}(\mathbf{x}) \mathrm{d} \mathbf{x}
$$

For example, one may cover the computational domain with a uniform rectangular mesh and use the midpoint rule, in which case $D_{i}(0)=\left[x_{i}^{p}(0)-\frac{\Delta x}{2}, x_{i}^{p}(0)+\frac{\Delta x}{2}\right] \times\left[y_{i}^{p}(0)-\frac{\Delta y}{2}, y_{i}^{p}(0)+\frac{\Delta y}{2}\right]$ and

$$
\alpha_{i}(0):=\Delta x \Delta y \varphi_{0}\left(\mathbf{x}_{i}^{p}(0)\right) .
$$

In general, the system (2.19) has to be solved numerically, and at final time $t_{\text {fin }}$, the solution $\varphi\left(\mathbf{x}, t_{\text {fin }}\right)$ should be recovered from the computed particle approximation $\varphi_{N}\left(\mathbf{x}, t_{\mathrm{fin}}\right)$. The reconstruction procedure, which is one of the key issues of this paper, is discussed in detail in the next section. 


\subsection{The finite-volume-particle method}

The finite-volume-particle (FVP) method for (1.1)-(1.2) is a hybrid of the methods in Sections 2.1 and 2.2: we apply the semi-discrete central-upwind scheme to the Saint-Venant system (1.1), while the transport equation (1.2) is solved by the particle method. We now present a detailed description of the method.

Following [14], we start by rewriting the system (1.1) in terms of the water surface, $w:=h+B$, and the $x$ and $y$-discharges, $h u$ and $h v$,

$$
\left\{\begin{array}{l}
w_{t}+(h u)_{x}+(h v)_{y}=S \\
(h u)_{t}+\left[\frac{(h u)^{2}}{w-B}+\frac{g}{2}(w-B)^{2}\right]_{x}+\left[\frac{(h u)(h v)}{w-B}\right]_{y}=-g(w-B) B_{x} \\
(h v)_{t}+\left[\frac{(h u)(h v)}{w-B}\right]_{x}+\left[\frac{(h v)^{2}}{w-B}+\frac{g}{2}(w-B)^{2}\right]_{y}=-g(w-B) B_{y}
\end{array}\right.
$$

The central-upwind scheme (2.9)-(2.15) is then applied to this system. To this end, the quadratures for $\bar{R}_{j, k}(t):=\left(\bar{R}_{j, k}^{(1)}(t), \bar{R}_{j, k}^{(2)}(t), \bar{R}_{j, k}^{(3)}(t)\right)^{T}$ that appear on the right-hand side (RHS) of (2.15) should be specified. The quadrature in $\bar{R}_{j, k}^{(1)}$ depends on the type of the source $S$. For instance, if the source is a smooth function as in Section 3, Example 3, the midpoint rule can be used:

$$
\bar{R}_{j, k}^{(1)}(t)=S\left(x_{j}, y_{k}, t\right)
$$

A simpler example of a spatially localized point source is considered in [5]. To compute $\bar{R}_{j, k}^{(2)}$ and $\bar{R}_{j, k}^{(3)}$, one should use the special quadrature,

$$
\begin{aligned}
& \bar{R}_{j, k}^{(2)}(t)=-\frac{g}{2}\left(w_{j, k}^{\mathrm{E}}-B\left(x_{j+\frac{1}{2}}, y_{k}\right)+w_{j, k}^{\mathrm{W}}-B\left(x_{j-\frac{1}{2}}, y_{k}\right)\right) \cdot \frac{B\left(x_{j+\frac{1}{2}}, y_{k}\right)-B\left(x_{j-\frac{1}{2}}, y_{k}\right)}{\Delta x} \\
& \bar{R}_{j, k}^{(3)}(t)=-\frac{g}{2}\left(w_{j, k}^{\mathrm{N}}-B\left(x_{j}, y_{k+\frac{1}{2}}\right)+w_{j, k}^{\mathrm{S}}-B\left(x_{j}, y_{k-\frac{1}{2}}\right)\right) \cdot \frac{B\left(x_{j}, y_{k+\frac{1}{2}}\right)-B\left(x_{j}, y_{k-\frac{1}{2}}\right)}{\Delta y}
\end{aligned}
$$

where, as before, $w_{j, k}^{\mathrm{E}(\mathrm{W}, \mathrm{N}, \mathrm{S})}$ denote the corresponding point values of the piecewise linear reconstruction of $w$, see (2.12). Using quadrature (2.22) guarantees the preservation of the stationary steady-state solution $(w \equiv$ Const, $\left.(h u, h v)^{T} \equiv 0, S \equiv 0\right)$, which corresponds to the "lake at rest" state. This property is especially important when quasi-stationary solutions are concerned (see $[1,14]$ for details).

In our FVP method, we will apply the central-upwind scheme to the Saint-Venant system (2.21) only, but it can be applied to the transport equation (1.2) as well. In the latter case, the numerical dissipation present at the central-upwind scheme can be reduce by applying it to the system (2.21) and equation (1.2) separately, as described in Appendix B. This approach leads to a reasonable overall resolution, as demonstrated in the numerical experiments in Section 3 and in [5]. However, the contact waves in pollution concentration are still excessively smeared, and therefore it would be desirable to further reduce the amount of numerical dissipation.

In order to achieve this goal, we solve equation (1.2) using the aforementioned particle method with $\varphi:=h T$, $\xi:=(u, v)^{T}$, and $f:=T_{S} S$. Since the Saint-Venant system (1.1) can be solved independently of the transport equation (1.2), the particle method, described in Section 2.2, can be applied directly to (1.2). To do that, one will need to know the values of the velocity vector $\left(u\left(\mathbf{x}_{i}^{p}(t), t\right), v\left(\mathbf{x}_{i}^{p}(t), t\right)\right)^{T}$ and the functions $\beta_{i}$ in $(2.19)$. The velocities, together with their partial derivatives required in (2.20), can be calculated from the piecewise linear reconstruction of $w, h u$, and $h v$, used for solving (2.21) by the central-upwind scheme. The functions $\beta_{i}$ depend on the type of the source term in (1.2). A particular example is considered in Section 3, Example 3. 
Remark. Notice that, we use two different grids in our hybrid method. The grid for the central-upwind scheme, $\left\{\left(x_{j}, y_{k}\right)\right\}$, is fixed, while the particles locations, $\left\{\mathbf{x}_{i}^{p}(t)\right\}$, change in time according to the flow velocity.

Recall that after applying the particle method to equation (1.2), only the locations $\left\{\mathbf{x}_{i}^{p}\left(t_{\text {fin }}\right)\right\}$ of the particles and their weights $\left\{\alpha_{i}\left(t_{\text {fin }}\right)\right\}$ will be available, and point values of the computed solution are to be recovered from the particle distribution. A commonly used way of such a reconstruction is to regularize the particle solution,

$$
(h T)_{N}(\mathbf{x}, t)=\sum_{i=1}^{N} \alpha_{i}(t) \delta\left(\mathbf{x}-\mathbf{x}_{i}^{p}(t)\right),
$$

by taking a convolution product with a smooth kernel $\zeta_{\varepsilon}(x)$, namely,

$$
(h T)_{N}^{\varepsilon}(\mathbf{x}, t)=\sum_{i=1}^{N} \alpha_{i}(t) \zeta_{\varepsilon}\left(\mathbf{x}-\mathbf{x}_{i}^{p}(t)\right),
$$

where $\zeta_{\varepsilon}$ serves as a smooth approximation of the $\delta$-function satisfying

$$
\zeta_{\varepsilon}=\frac{1}{\varepsilon^{2}} \zeta\left(\frac{\mathbf{x}}{\varepsilon}\right), \quad \iint_{\mathbb{R}^{2}} \zeta(\mathbf{x}) \mathrm{d} \mathbf{x}=1 .
$$

It is well-known that the quality of this approximation depends on the smoothness of the solution [22], and, unfortunately, when applied to discontinuous solutions, this reconstruction procedure will either smear the discontinuities or lead to oscillations, depending on the value of $\varepsilon$, as illustrated in Section 3, Example 1, Figure 3.2. In this 1-D example, Gaussian kernel $\zeta(x)=\frac{1}{\sqrt{\pi}} \mathrm{e}^{-x^{2}}$ was used, but other smooth kernels give similar results.

To overcome this difficulty, in [5], where the 1-D version of the problem (1.1)-(1.2) was studied, the computed particle data were interpreted as integrals of the approximated solution over some intervals around the particles. Then, dividing the weights $\alpha_{i}$ by the lengths of the corresponding intervals, the cell averages $(\overline{h T})_{i}$ were obtained. Since the resulting piecewise constant approximation may be very oscillatory, a nonlinear filter from [7] was implemented as a post-processing. However, even in the 1-D case, such an approach is of a limited use, as it is demonstrated in Section 3, Example 1. Furthermore, the key drawback of this technique is that its 2-D generalization is rather problematic since particles, moving with the flow, can form a very complicated unstructured grid.

In this paper, we propose a new method that allows to completely avoid any loss of resolution attributed to the regularization of the particle distribution, and at the same time, does not produce any spurious oscillations. The idea is to consider an equation on pollutant concentration $T$,

$$
T_{t}+u T_{x}+v T_{y}=\frac{\left(T_{S}-T\right) S}{h}
$$

which is dual to equation (1.2). Multiplying equation (1.2) by $T$ and equation (2.25) by $h T$, adding them up, and integrating with respect to $\mathbf{x}$, we obtain

$$
\frac{\mathrm{d}}{\mathrm{d} t} \int_{\mathbb{R}^{2}}(h T) T \mathrm{~d} \mathbf{x}=\iint_{\mathbb{R}^{2}} h T \frac{\left(2 T_{S}-T\right) S}{h} \mathrm{~d} \mathbf{x},
$$

provided either $T$ or $(h u, h v)$ vanish at infinity.

Let us now take $(h T)(\mathbf{x}, 0)$ to be a single particle, $\alpha_{i}(0) \delta\left(\mathbf{x}-\mathbf{x}_{i}(0)\right)$, which, as it is explained above, will evolve in time according to the ODE system (2.19). Then equation (2.26) reduces to the single ODE along the 
generalized characteristic $\mathbf{x}_{i}(t)$ :

$$
\frac{\mathrm{d}}{\mathrm{d} t}\left[(\alpha T)\left(\mathbf{x}_{i}(t), t\right)\right]=\frac{\left\{2 T_{S} \alpha_{i}(t)-(\alpha T)\left(\mathbf{x}_{i}(t), t\right)\right\} S\left(\mathbf{x}_{i}(t), t\right)}{h\left(\mathbf{x}_{i}(t), t\right)} .
$$

Notice that since both $h$ and $S$ may be discontinuous, the RHS of (2.27) may be discontinuous as well. However, as long as $S$ is bounded and $h \geq$ Const $>0$, the RHS of (2.27) is bounded, and thus the existence of its generalized solution is guaranteed by the theory of Filippov [8-10]. In practice, $h$ is a piecewise smooth function with a finite number of discontinuities only, and therefore one can numerically solve (2.27) by the same ODE solver used to solve the system (2.19). While doing this, $h\left(\mathbf{x}_{i}(t), t\right)$ is replaced with $\widetilde{w}\left(\mathbf{x}_{i}(t), t\right)-B\left(\mathbf{x}_{i}(t)\right)$, where $\widetilde{w}$ is a piecewise linear reconstruction of $w$, used for solving $(2.21)$ by the central-upwind scheme.

Finally, at time $t=t_{\text {fin }}$ the point values of $T$ are obtained by

$$
T\left(\mathbf{x}_{i}\left(t_{\text {fin }}\right), t_{\text {fin }}\right)=\frac{(\alpha T)\left(\mathbf{x}_{i}\left(t_{\text {fin }}\right), t_{\text {fin }}\right)}{\alpha_{i}\left(t_{\text {fin }}\right)} .
$$

\section{Remarks.}

1. Notice that equation (2.25) is a consequence of (1.2) and of the first equation of (1.1) (at least for smooth solutions).

2. If $S \equiv 0$, then $\alpha_{i}(t) \equiv \alpha_{i}(0)$ and $(2.27)$ becomes trivial, and its solution is

$$
T\left(\mathbf{x}_{i}\left(t_{\text {fin }}\right), t_{\text {fin }}\right)=T\left(\mathbf{x}_{i}(0), 0\right) .
$$

Note that in this case, the particle method for $h T$ reduces to the method of characteristics for $T$. However, one can verify that this will no longer be true if the RHS of (2.27) is not zero, that is, if $S \not \equiv 0$.

In Appendix A, we illustrate an advantage of our approach over the method of characteristics on an example with a point source, where the method of characteristics fails while the FVP method produces an accurate solution.

3. We would like to point out that the first component of the presented hybrid FVP method, the Godunov type central-upwind scheme, can be replaced with one's favorite finite-volume method for the Saint-Venant system (1.1).

\section{Numerical ExAmples}

In this section, we illustrate the performance of the FVP method in a number of 1-D and 2-D numerical examples. We also compare these results with the corresponding solutions computed by the semi-discrete central-upwind finite-volume (FV) scheme, applied to both (2.21) and (1.2) with a separate estimates of the local speeds, as it is described in Appendix B. In all the examples, the gravity constant was taken $g=9.8$, the piecewise linear interpolants were constructed using the minmod limiter $(2.6)-(2.7)$ with $\theta=1.2$, the particles were initially uniformly distributed, and the arising systems of ODEs were solved by the third-order SSP Runge-Kutta method [13].

Example 1 (1-D dam break). This example is devoted to the 1-D case, in which the Saint-Venant system (1.1) reduces to

$$
\left\{\begin{array}{l}
h_{t}+(h u)_{x}=S \\
(h u)_{t}+\left(h u^{2}+\frac{g}{2} h^{2}\right)_{x}=-g h B^{\prime}(x),
\end{array}\right.
$$

and the transport equation (1.2) becomes

$$
(h T)_{t}+(u h T)_{x}=T_{S} S .
$$


(a)

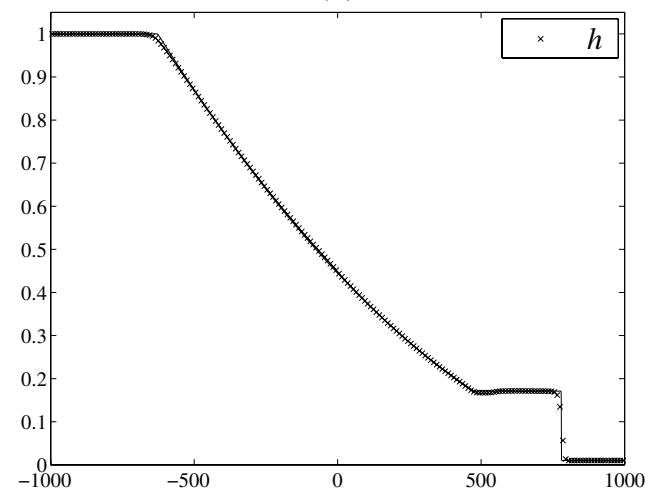

(c)

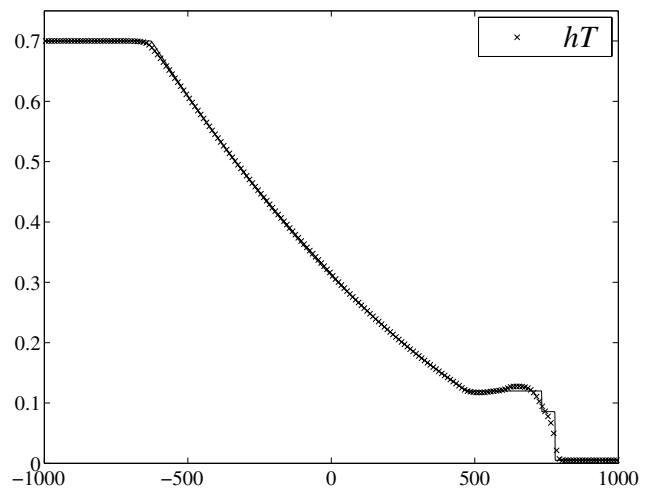

(b)

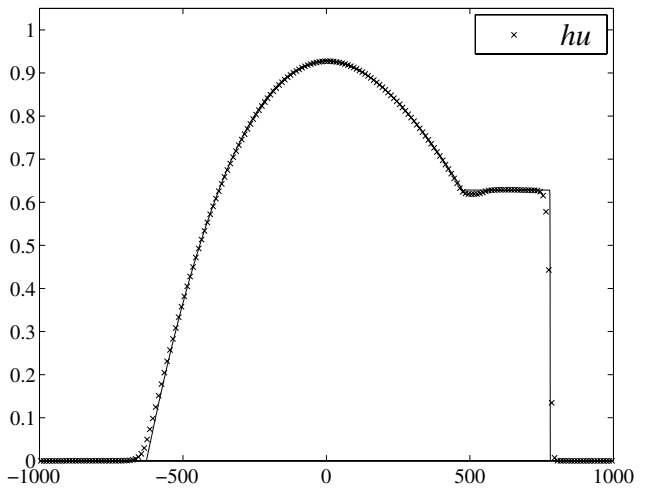

(d)

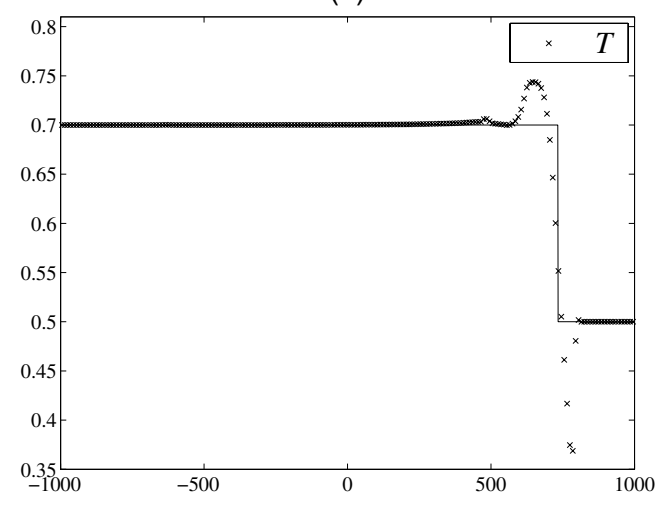

Figure 3.1. $h(\mathrm{a}), h u(\mathrm{~b}), h T(\mathrm{c})$, and $T(\mathrm{~d})$ by the FV method.

For the detailed description of the 1-D FVP method we refer the reader to [5], and the 1-D semi-discrete central-upwind scheme can be found in [15-17].

A particular set of the data (taken from [2]) corresponds to a dam break on a flat bottom $(B \equiv 0)$ in the domain $[-1000,1000]$, where the pollutant has different concentrations on each side of the dam. The initial Riemann data are:

$$
(h, u, T)(x, 0)= \begin{cases}(1.00,0,0.7), & \text { if } x<0, \\ (0.01,0,0.5), & \text { if } x>0,\end{cases}
$$

and there is no source, that is, $S \equiv 0$.

We apply the FVP and FV methods with $\Delta x=10$ for both the central-upwind scheme and the initial distribution of the particles. The results at time $t_{\text {fin }}=200$ are presented in Figures 3.1-3.3. In Figures 3.1a-d, we show the values of the evolved quantities $h, h u, h T$, and the pollutant concentration $T:=h T / h$, respectively, obtained by the FV method.

In Figures 3.2 and 3.3, we compare the values of $h T$ and $T$, obtained by applying different versions of the FVP method. In all the cases, the locations of particles at the final time, $\left\{x_{i}^{p}\left(t_{\mathrm{fin}}\right)\right\}$, are computed by numerical integration of the ODE system (2.19) with $\xi=u$ and $\beta_{i}(t) \equiv 0$ implying, in particular, that $\alpha_{i}(t) \equiv \alpha_{i}(0)$ for all $t>0$.

First, we recover the point values of $h T$ by a regularization procedure according to (2.23)-(2.24) with $\zeta_{\varepsilon}(x)=\frac{1}{\sqrt{\pi} \varepsilon} \mathrm{e}^{-x^{2} / \varepsilon^{2}}$ and $\varepsilon=20 \sqrt{\Delta x}$, and compute the point values of $T$ using the following formula

$$
T\left(x_{i}^{p}\left(t_{\mathrm{fin}}\right), t_{\mathrm{fin}}\right)=\frac{(h T)\left(x_{i}^{p}\left(t_{\mathrm{fin}}\right), t_{\mathrm{fin}}\right)}{h\left(x_{i}^{p}\left(t_{\mathrm{fin}}\right), t_{\mathrm{fin}}\right)},
$$


(a)

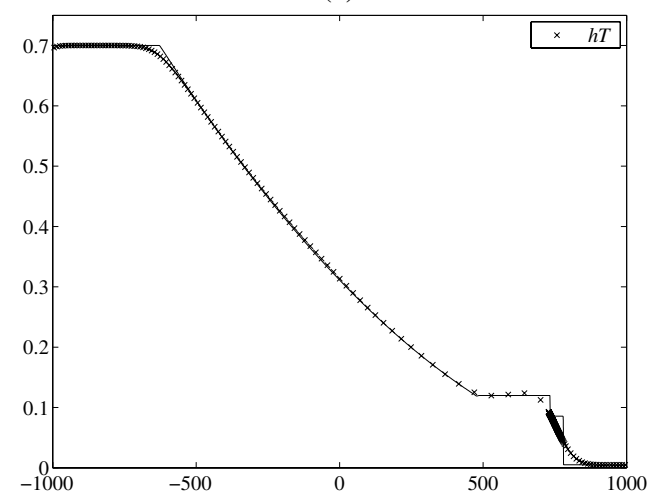

(b)

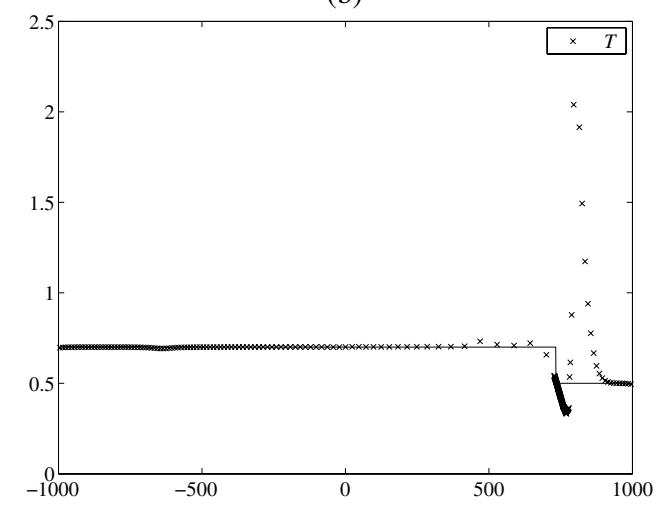

FiguRE 3.2. $h T$ and $T$ obtained by the FVP method with regularization $(2.23),(2.24)$.

where $h\left(x_{i}^{p}\left(t_{\text {fin }}\right), t_{\text {fin }}\right)$ are the corresponding values of the reconstructed piecewise linear interpolant

$$
\widetilde{h}\left(x, t_{\text {fin }}\right)=\bar{h}_{j}\left(t_{\text {fin }}\right)+\left(h_{x}\right)_{j}\left(x-x_{j}\right), \quad x_{j-\frac{1}{2}}<x<x_{j+\frac{1}{2}} .
$$

The resulting $h T$ and $T$ are plotted in Figure 3.2. One can clearly observe a poor resolution of discontinuities of the computed solution.

Second, we implement the reconstruction method introduced in [5]: the weights $\alpha_{i}(t) \equiv \alpha_{i}(0)$ are interpreted as integrals of the approximated solution over some intervals around the particles and the point values of $h T$ are computed by dividing the $\alpha_{i}(0)$ by the lengths of the corresponding intervals, namely,

$$
(h T)\left(x_{i}^{p}\left(t_{\mathrm{fin}}\right), t_{\mathrm{fin}}\right)=\frac{2 \alpha_{i}(0)}{x_{i+1}^{p}\left(t_{\mathrm{fin}}\right)-x_{i-1}^{p}\left(t_{\mathrm{fin}}\right)} .
$$

The point values of $T$ are obtained, as before, from (3.4)-(3.5). The results are shown in Figures 3.3a-b, where one can see very large oscillations both near the contact discontinuity and around the shock.

We then post-process these data by applying a nonlinear filter [7] to both $h T$ and $T$ (the data are first projected onto the uniform grid, see [5] for the details). As in the examples, presented in [5], the filter manages to remove the oscillations, see Figures $3.3 \mathrm{c}-\mathrm{d}$, but the achieved resolution is not as good as in the dam break example in [5], where we considered the same problem with a slightly different initial data:

$$
(h, u, T)(x, 0)= \begin{cases}(1.0,0,0.7), & \text { if } x<0 \\ (0.5,0,0.5), & \text { if } x>0\end{cases}
$$

instead of (3.3). It should be also pointed out that even though the nonlinear filter is a very powerful tool for removing spurious oscillations, it may sometimes average out physically important features of the solution (especially in the 2-D case) and to produce a "staircase-like" approximations as in Figures 3.3c-d.

Finally, we implement the new version of the FVP method, presented in this paper. The computed pollutant concentration, $T$, is shown in Figure 3.3f. As one can clearly see, the achieved resolution is almost perfect. As for the computed values of $h T$, presented in Figure 3.3e, the accuracy obviously deteriorate in the vicinity of the shock, since $h T$ is obtained by multiplying the computed values of $T$ and $h$, and the $L_{\text {loc }}^{\infty}$-error in $h$ is $\mathcal{O}(1)$ there. 
(a)

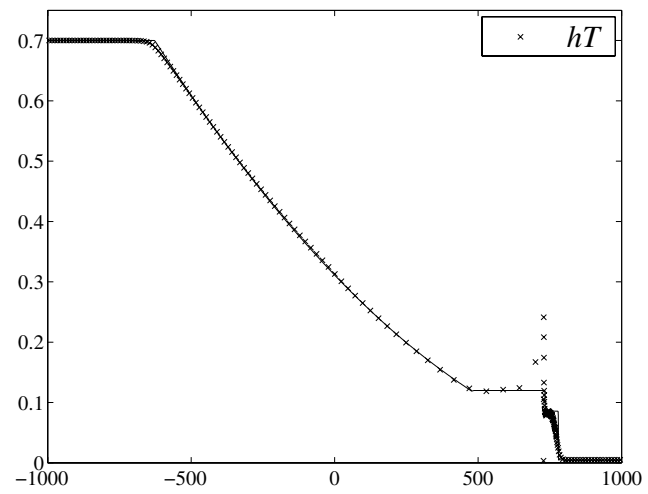

(c)

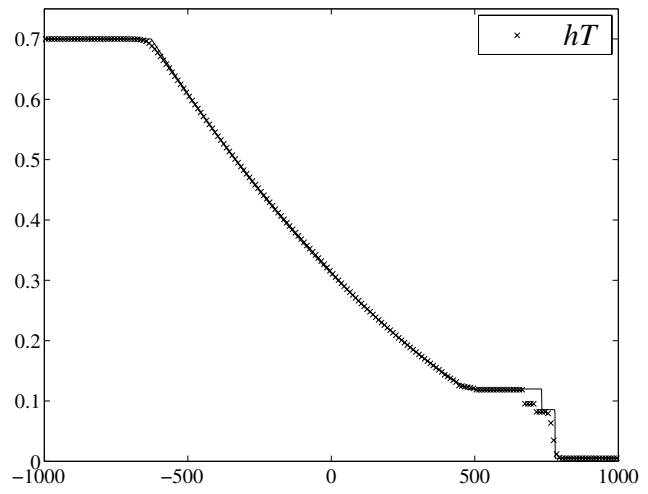

(e)

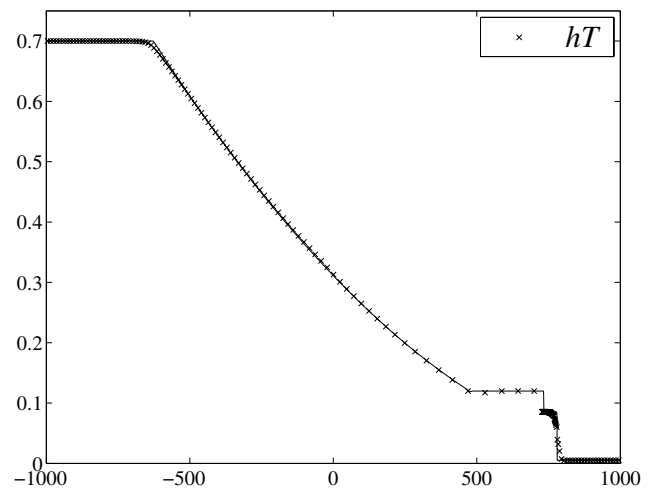

(b)

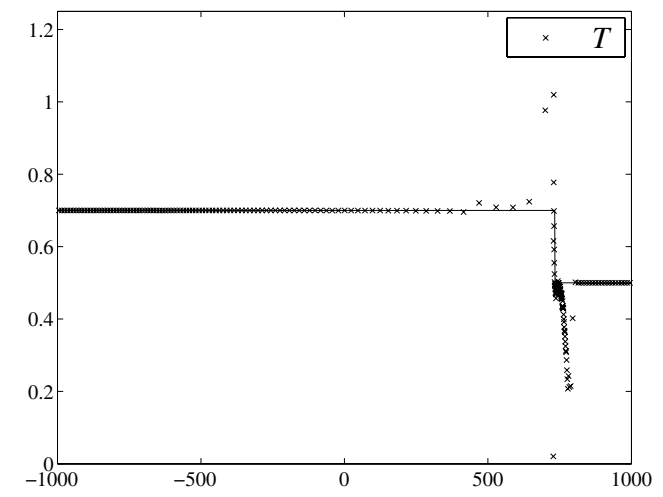

(d)

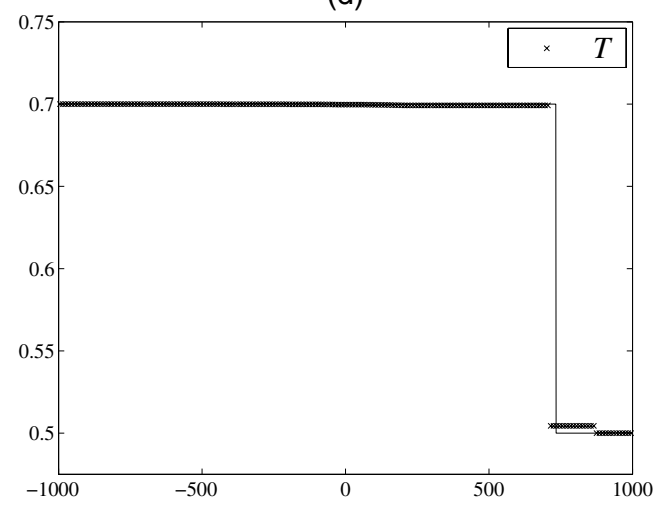

(f)

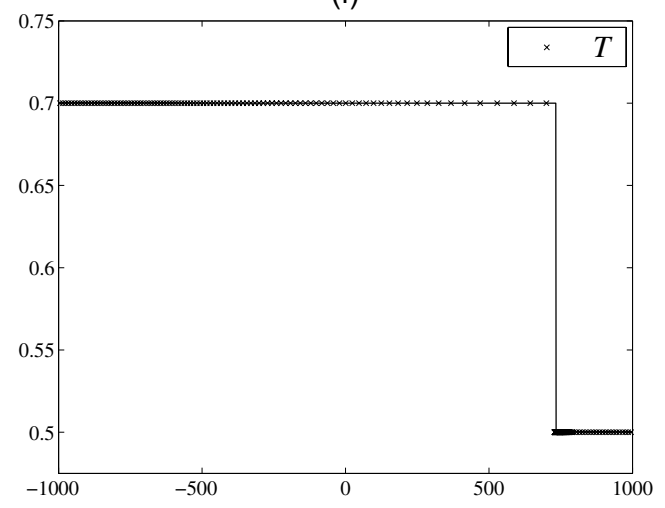

FIgure 3.3. $h T$ and $T$ obtained by the three different versions of the FVP method: (a) and (b) - the FVP method from [5] without filtering; (c) and (d) - the FVP method from [5] with filtering; (e) and (f) - the new FVP method.

\section{Remarks.}

1. Notice that $h$ and $h u$ are computed by the same central-upwind scheme for both the FVP and FV methods, and therefore we plot them only once.

2. The reference solution is obtained by the new FVP method with $\Delta x=0.4$. 


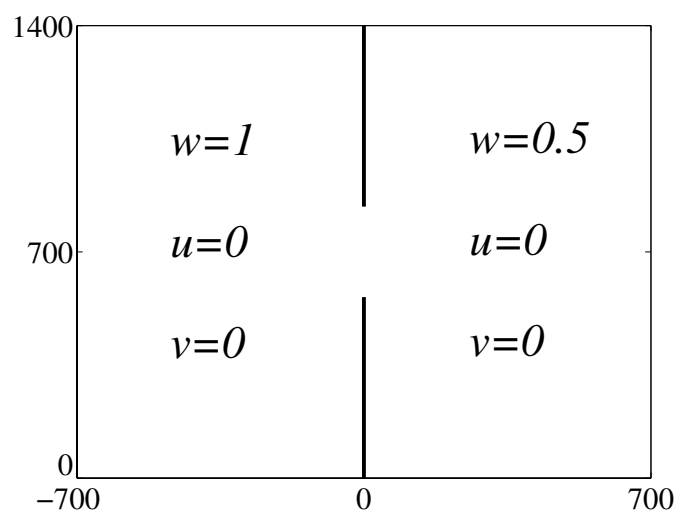

FIGURE 3.4. Initial setting in Example 2.
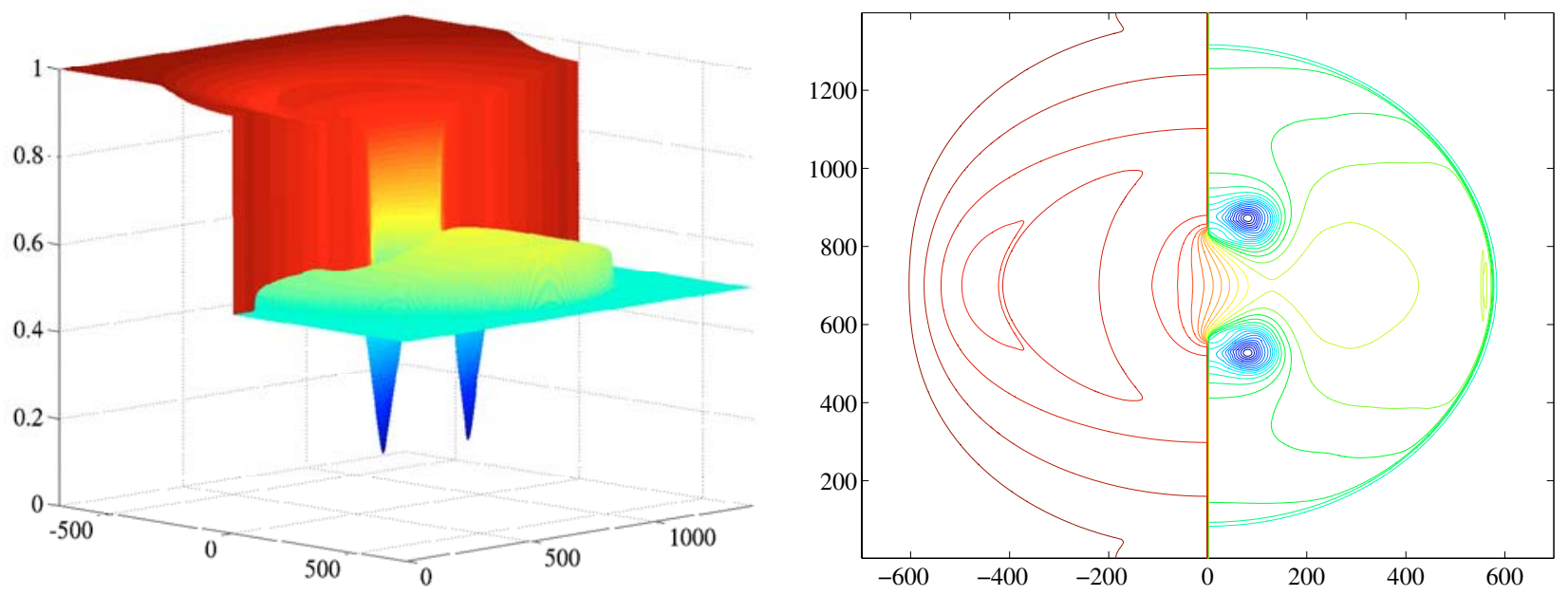

Figure 3.5. A 3-D plot (left) and a contour plot (right) of $h$.

Example 2 (2-D dam break on a flat bottom without source). In this example, we solve the 2-D system $(2.21),(1.2)$ in the square domain outlined in Figure 3.4. We consider the case of a dam break on a flat bottom $(B \equiv 0)$ without source $(S \equiv 0)$. The initial concentration of pollutant is

$$
T(x, y, 0)=\left\{\begin{array}{cl}
\mathrm{e}^{-0.0001\left[(x+50)^{2}+(y-600)^{2}\right]}, & \text { if }-700 \leq x<0,0 \leq y \leq 1400 \\
0.5, & \text { if } 0 \leq x \leq 700,0 \leq y \leq 1400
\end{array}\right.
$$

and the rest of the initial data is shown in Figure 3.4.

The water flows through the breach, located between $y=560$ and $y=840$, from the left to the right. The solution computed on a $500 \times 500$ grid at time $t_{\text {fin }}=200$ is shown in Figures 3.5-3.7. The water height $h$, computed by the same second-order central-upwind scheme (2.9)-(2.15) for both the FV and FVP methods, is presented in Figure 3.5. As one can clearly see, the scheme provides a very high resolution of both the circular shock wave and the vortices formed on the both sides of the breach. In Figures 3.6-3.7, we show the pollutant concentration $T$, computed by the FV and FVP methods. One can observe a better resolution of the fronts, and especially of the structures inside the vortices, achieved by the FVP method, see Figures 3.6-3.7 (right). 

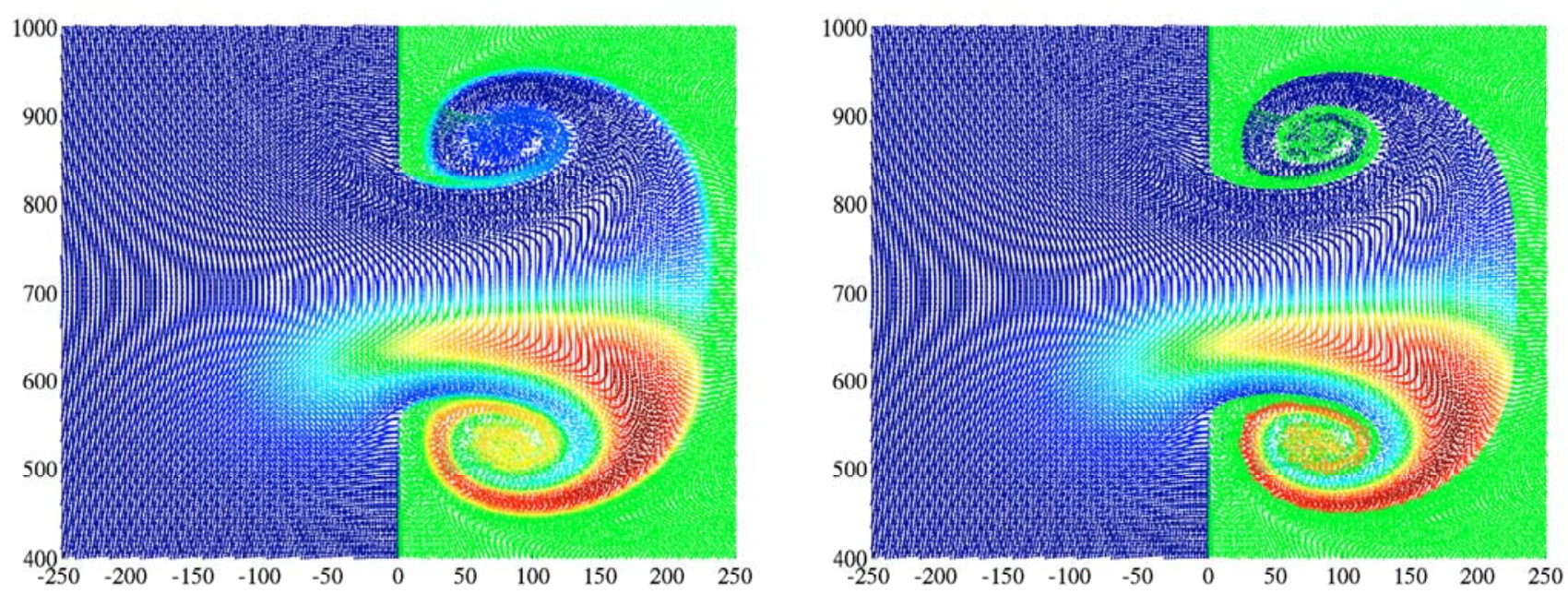

Figure 3.6. Top view on $T$ computed by the FV (left) and FVP (right) methods.
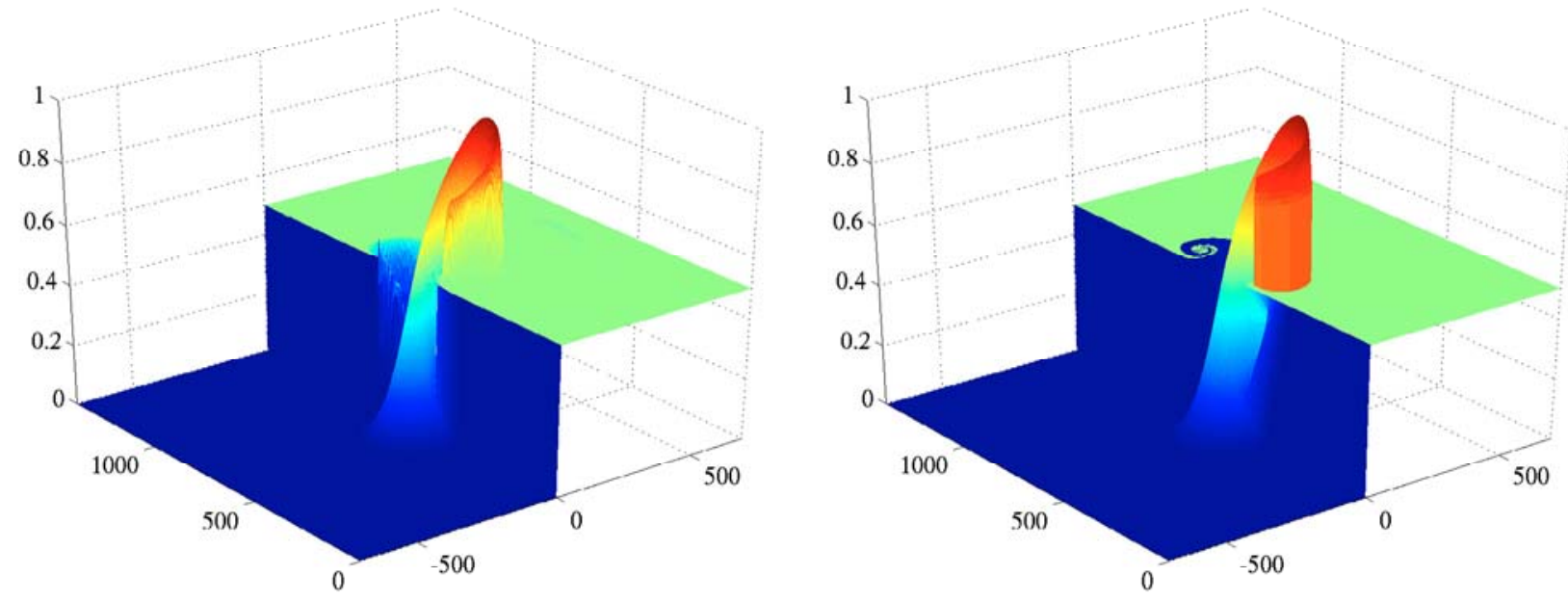

Figure 3.7. $T$ computed by the FV (left) and FVP (right) methods.

In addition, we would like to recall that, while applying the FVP method, one does not need to calculate the values of $T$ at every time step, since $S \equiv 0$ for this problem. Once the location of the particles are computed at final time $t_{\text {fin }}, T\left(\mathbf{x}_{i}\left(t_{\text {fin }}\right), t_{\text {fin }}\right)=T\left(\mathbf{x}_{i}(0), 0\right)$, according to $(2.28)$.

Example 3 (2-D dam break on a nonflat bottom with nonzero source). We consider the 2-D system (2.21), (1.2) in the square domain $[-300,1100] \times[0,1400]$ subject to the initial data shown in Figure 3.8, where the shape of the dam is given by

$$
\Gamma(y)= \begin{cases}\min \left[200+\frac{(y-700)^{2}}{400}, 600\right], & \text { if } 0 \leq y<700, \\ 200, & \text { if } 700 \leq y \leq 1400 .\end{cases}
$$




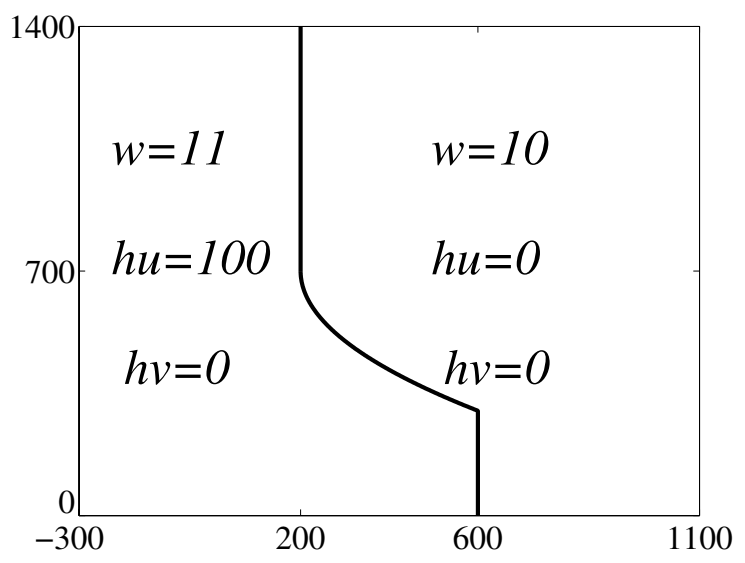

FIGURE 3.8. Initial setting in Example 3.

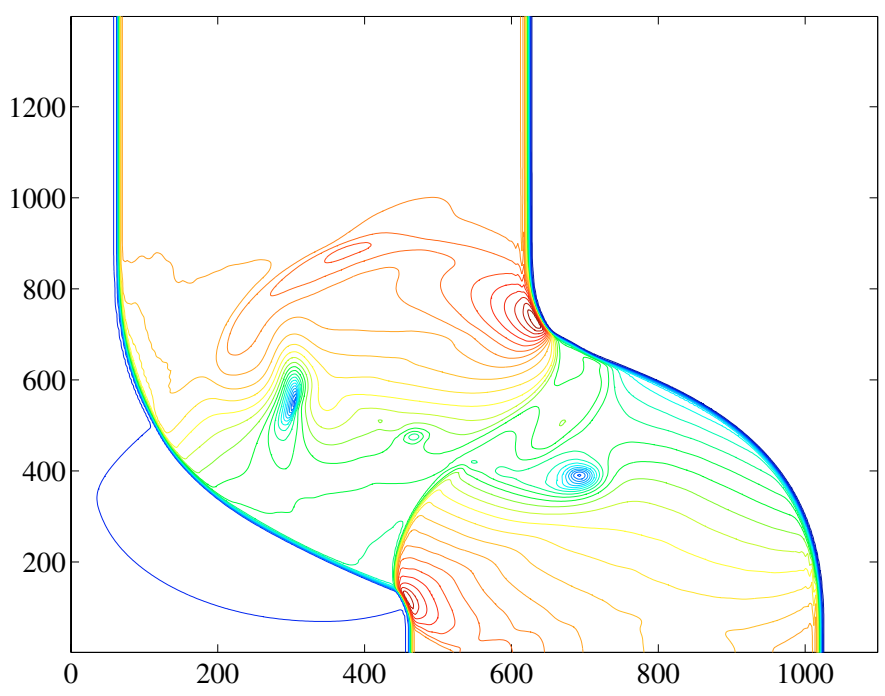

Figure 3.9. A contour plot of $w$.

In this example, the bottom consists of three elliptic-shape exponential humps:

$$
B(x, y)=4.5\left[\mathrm{e}^{-\kappa_{1}(x-500)^{2}-\kappa_{2}(y-700)^{2}}+\mathrm{e}^{-\kappa_{2}(x-300)^{2}-\kappa_{1}(y-600)^{2}}+\mathrm{e}^{-\kappa_{2}(x-700)^{2}-\kappa_{1}(y-700)^{2}}\right],
$$

with $\kappa_{1}=10^{-4}$ and $\kappa_{2}=10^{-3}$. We assume that initially the water is practically clean, that is $T(x, y, 0) \sim 0$, but later on a source of polluted water with the concentration of pollutant $T_{s}=25$ is turned on:

$$
S(x, y, t)=0.5 \mathrm{e}^{-0.5(t-8)^{2}-0.00001(x+y-1000)^{2}-0.0005(x-y+200)^{2}} .
$$

The solutions at time $t_{\text {fin }}=30$, computed by the FV and FVP methods on a $500 \times 500$ grid, are shown in Figures 3.9-3.12. An interaction of the initially curved shock wave with a complicated nonflat bottom results in a rather complicated wave structures, see Figure 3.9. The time evolution of the pollutant is tracked in Figure 3.10. 
(a)

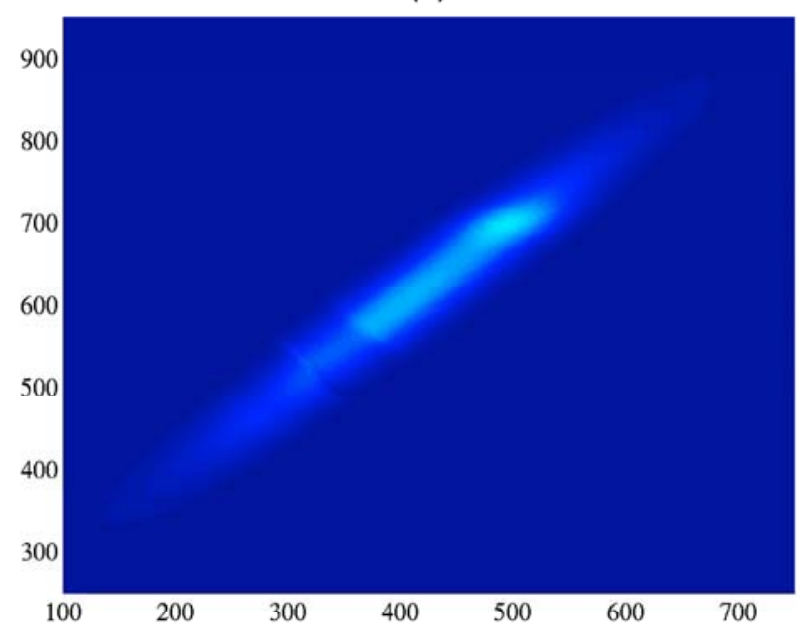

(c)

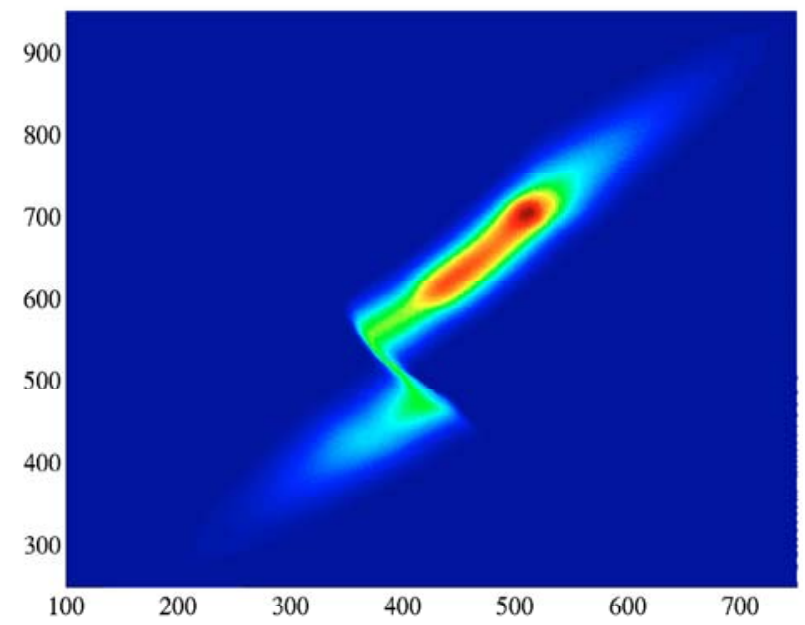

(b)

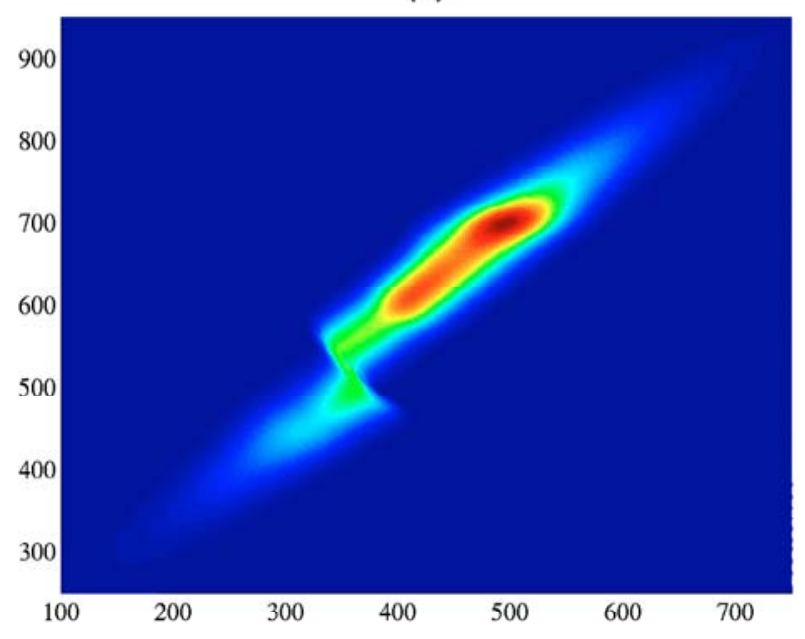

(d)

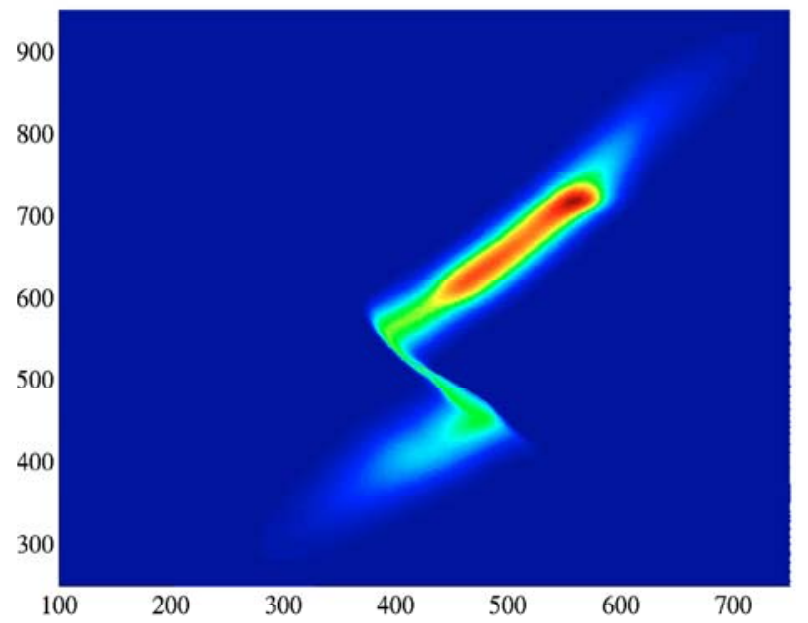

Figure 3.10. Top view on $T$ at $t=7.5$ (a), 15 (b), 22.5 (c), and 30 (d) computed by the FVP method.

In order to compare the FV and the FVP methods, we present the three-dimensional (3-D) plots of the pollutant concentration $T$ in Figure 3.11. As one can observe, the FVP method outperforms the FV method (it can be especially clearly seen in the 2-D projection shown in Fig. 3.12).

Acknowledgements. The authors are grateful to Eitan Tadmor for a breakthrough suggestion to consider the dual equation. The work of A. Chertock was supported in part by the NSF Grant \# DMS-0410023. The research of A. Kurganov was supported in part by the NSF Grant \# DMS-0310585. A part of this research was conducted at the Institute of Pure and Applied Mathematics, University of California Los Angeles, where both authors participated in the Modern Applied Mathematics for the Atmospheric and Oceanic Sciences Summer School (July 14-August 2, 2003). 

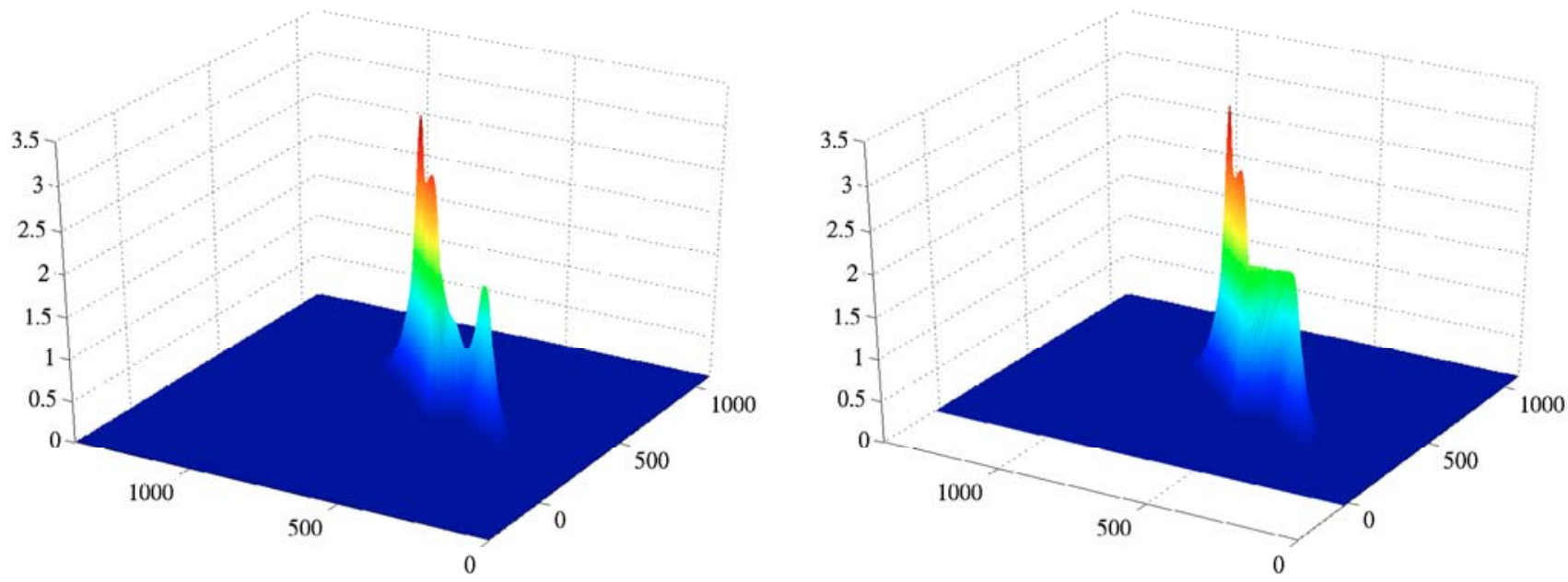

Figure 3.11. T computed by the FV (left) and FVP (right) methods.
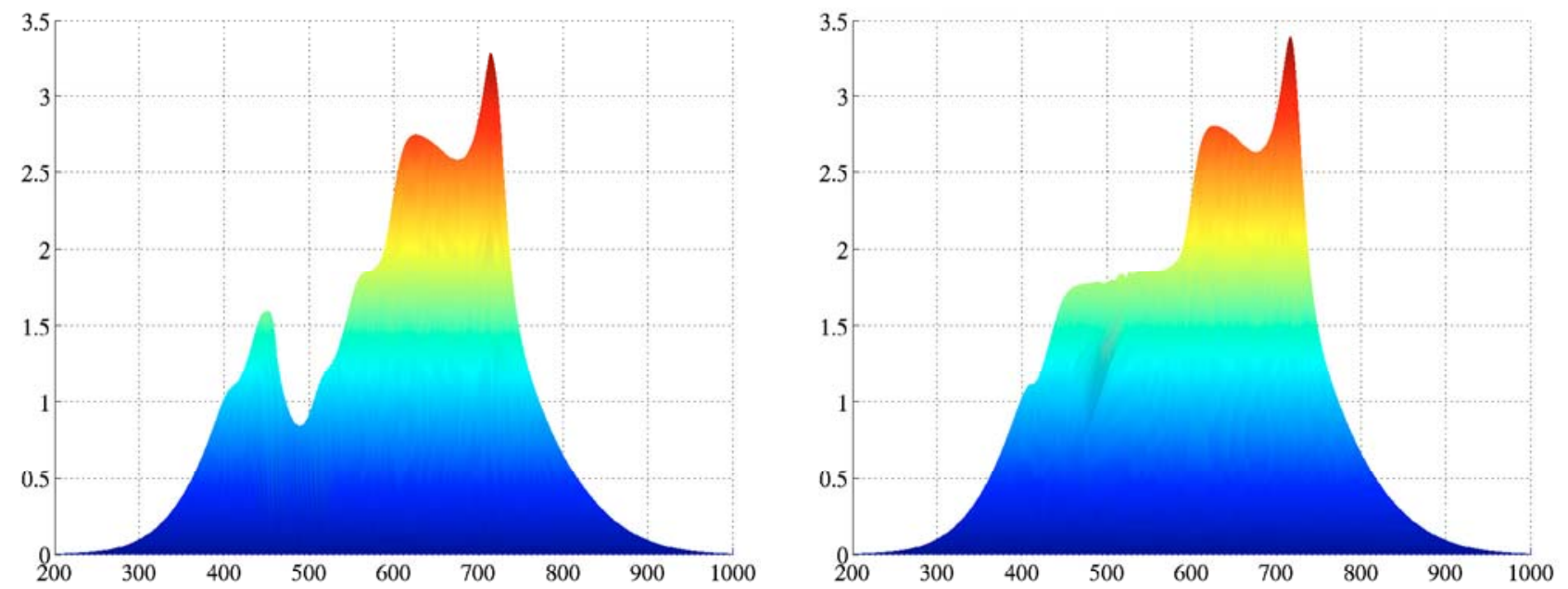

Figure 3.12. 2-D projections of $T$ computed by the FV (left) and FVP (right) methods.

\section{Appendix A}

Here, we bring a 1-D example, taken from [2], that illustrates an advantage of our method of computing the point values of $T$ over the method of characteristics, which can be also used for solving equation (2.25). The water flow is described by

$$
\left\{\begin{array}{l}
h_{t}+(h u)_{x}=S(x, t), \\
(h u)_{t}+\left(h u^{2}+\frac{h^{2}}{2}\right)_{x}=-h B^{\prime}(x),
\end{array}\right.
$$

where the point source, acting at $x=45$ between $t=100$ and $t=300$, is given by

$$
S(x, t)=0.01 \delta(x-45)[H(t-100)-H(t-300)],
$$


(a)

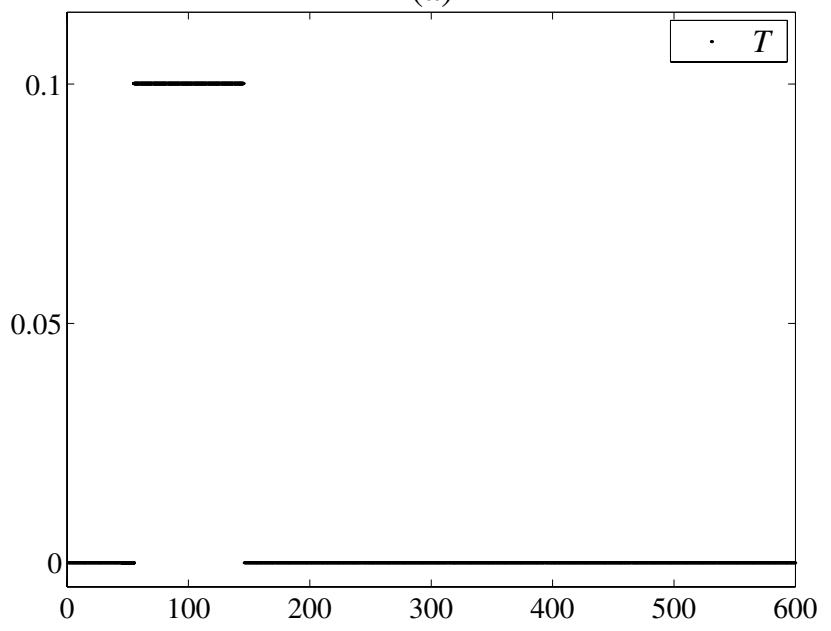

(b)

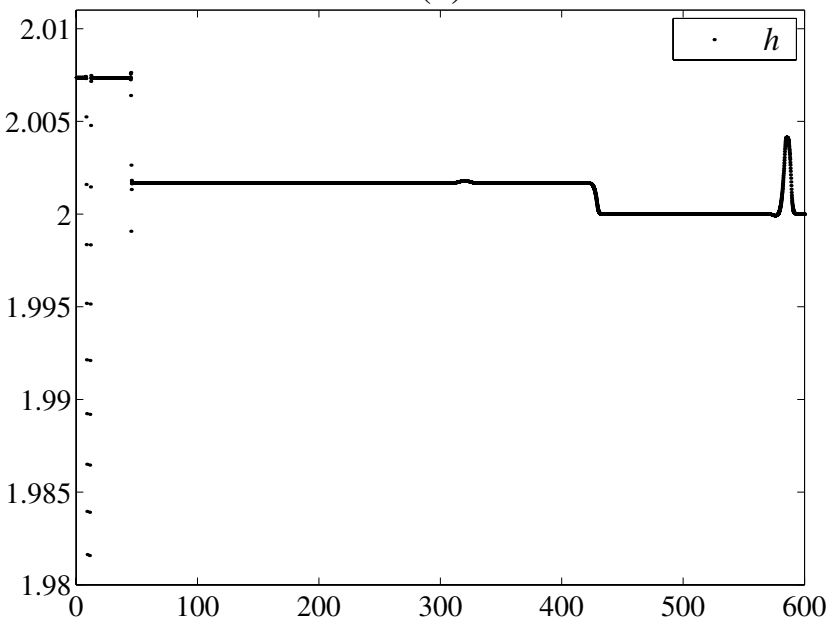

Figure A.1. $T$ (left) and $h$ (right) computed by the FVP method.

and the bottom topography is

$$
B(x)=\left(0.2-0.05(x-10)^{2}\right)[H(x-8)-H(x-12)] .
$$

Here and below, $H$ is a Heaviside function. The pollutant propagates according to the transport equation,

$$
(h T)_{t}+(u h T)_{x}=10 S,
$$

which can be (formally) rewritten in the advection form:

$$
T_{t}+u T_{x}=\frac{10-T}{h} S .
$$

The initial data for this example are: $h(x, 0)+B(x)=2, h(x, 0) u(x, 0)=1$, and $T(x, 0)=0$.

Solving this problem numerically requires a special treatment due to the presence of the point source on the RHS of the above equations. An implementation of the FVP method for the system (A.1), (A.4) has been presented in [5]. In this paper, we only show the computed solution at time $t=300$ (right after the pollution source has been turned off), see Figure A.1. Even though the exact solution of the problem is not available, these results agree well with those obtained by the two time step kinetic method [2] and by the central-upwind scheme [5], so that we can assume that the computed solution is sufficiently accurate. Therefore, in the following we will use the computed $T$ as a reference solution.

When the FVP method is applied in this example, the concentration $T$ is obtained by solving the following system of ODEs along each characteristic curve, $x=x_{i}^{p}(t)$ :

$$
\begin{aligned}
\frac{\mathrm{d} \alpha_{i}}{\mathrm{~d} t} & =0.01\left|D_{i}(t)\right| \delta\left(t-t_{i}^{*}\right), \\
\frac{\mathrm{d}(\alpha T)_{i}}{\mathrm{~d} t} & =\frac{20 \alpha_{i}(t)-(\alpha T)_{i}(t)}{h_{i}(t)} 0.01 \delta\left(t-t_{i}^{*}\right),
\end{aligned}
$$




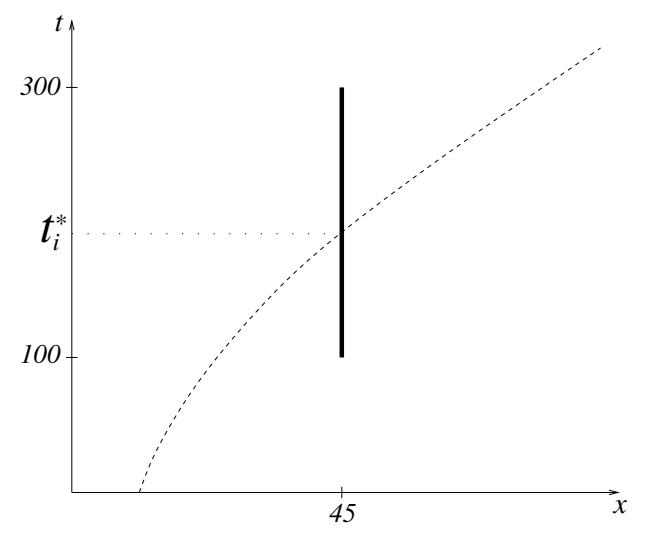

Figure A.2. The $i$ th characteristic in the $(x, t)$-plane.

where $x_{i}^{p}\left(t_{i}^{*}\right)=45$, see Figure A.2. Alternatively, one may solve equation (A.5) by the method of characteristics, which would require solving the ODE

$$
\frac{\mathrm{d} T_{i}}{\mathrm{~d} t}=\frac{10-T_{i}(t)}{h_{i}(t)} 0.01 \delta\left(t-t_{i}^{*}\right)
$$

along the $i$ th characteristic.

For a practical implementation, the delta-function on the RHS of both (A.7) and (A.8) is usually regularized. Following [5] we replace the delta-function with

$$
\delta_{\varepsilon}\left(t-t_{i}^{*}\right):=\frac{1}{\varepsilon}\left[H\left(t-t_{i}^{*}\right)-H\left(t-t_{i}^{*}-\varepsilon\right)\right]
$$

where $\varepsilon$ is proportional to $\Delta t$, and proceed to solving

$$
\frac{\mathrm{d} T_{i}}{\mathrm{~d} t}=\frac{10-T_{i}(t)}{h_{i}(t)} 0.01 \delta_{\varepsilon}\left(t-t_{i}^{*}\right)
$$

and

$$
\frac{\mathrm{d}(\alpha T)_{i}}{\mathrm{~d} t}=\frac{20 \alpha_{i}(t)-(\alpha T)_{i}(t)}{h_{i}(t)} 0.01 \delta_{\varepsilon}\left(t-t_{i}^{*}\right)
$$

together with (A.6) analytically. We start with the

Method of characteristics. We look for a solution of (A.9) in the form

$$
T_{i}(t)=\widehat{T}(t)+T_{i}\left(t_{i}^{*}+0\right) H\left(t-t_{i}^{*}\right)
$$

where we expect $\widehat{T}(t)$ to be continuous at $t=t_{i}^{*}$ and $\widehat{T}(t) \equiv 0$ for $t \leq t_{i}^{*}$. We also rewrite $h_{i}(t)$ in a similar manner:

$$
h_{i}(t)=\widehat{h}(t)+\left[h_{i}\left(t_{i}^{*}+0\right)-\widehat{h}_{i}\left(t_{i}^{*}\right)\right] H\left(t-t_{i}^{*}\right), \quad \widehat{h}_{i}(t) \text { is continuous at } t=t_{i}^{*} .
$$

Then, integrating (A.9) yields

$$
T_{i}(t)=\frac{1}{100 \varepsilon} \int_{t_{i}^{*}}^{t_{i}^{*}+\varepsilon} \frac{10-\widehat{T}(\tau)-T_{i}\left(t_{i}^{*}+0\right)}{\widehat{h}(\tau)+h_{i}\left(t_{i}^{*}+0\right)-\widehat{h}\left(t_{i}^{*}\right)} \mathrm{d} \tau, \quad t>t_{i}^{*}+\varepsilon
$$


Finally, assuming that both $\widehat{T}$ and $\widehat{h}$ are smooth on $\left(t_{i}^{*}, t_{i}^{*}+\varepsilon\right)$, we use their Taylor expansions about $t=t_{i}^{*}$ to conclude with

$$
T_{i}(t)=\frac{10-T_{i}\left(t_{i}^{*}+0\right)}{100 h_{i}\left(t_{i}^{*}+0\right)}+\mathcal{O}(\varepsilon), \quad t>t_{i}^{*}+\varepsilon .
$$

This implies (see Fig. A.1b for the relevant values of $h_{i}$ ) that

$$
T_{i}(t) \sim 0.05, \quad t>t_{i}^{*}+\varepsilon .
$$

The above computation clearly demonstrates (compare with Fig. A.1a) that in this particular example, the method of characteristics fails to provide an accurate approximation of the solution.

Next, we demonstrate how an accurate approximate solution is obtained by the

Particle method. We first integrate equation (A.6) to obtain

$$
\alpha_{i}(t) \equiv \alpha_{i}\left(t_{i}^{*}+0\right)=0.01\left|D_{i}\left(t_{i}^{*}\right)\right|, \quad t>t_{i}^{*} .
$$

We then solve equation (A.10) using the same approach as in the method of characteristics. This results in

$$
(\alpha T)_{i}(t)=\frac{20 \alpha_{i}\left(t_{i}^{*}+0\right)-(\alpha T)_{i}\left(t_{i}^{*}+0\right)}{100 h_{i}\left(t_{i}^{*}+0\right)}+\mathcal{O}(\varepsilon), \quad t>t_{i}^{*}+\varepsilon
$$

Solving the last equation for $(\alpha T)_{i}(t)$ and taking into account (A.12) and the data in Figure A.1b, we conclude with

which agrees with our reference solution in Figure A.1a.

$$
T_{i}(t):=\frac{(\alpha T)_{i}(t)}{\alpha_{i}(t)} \sim 0.1, \quad t>t_{i}^{*}+\varepsilon
$$

\section{Appendix B}

Here, we discuss an important detail related to the application of the central-upwind scheme to both (2.21) and (1.2) (in Sect. 3, this scheme has been referred to as the FV method). In principle, the central-upwind scheme described in Section 2.1 can be applied to the extended system (2.21), (1.2) in a straightforward manner. However, it is possible to reduce numerical dissipation by taking an advantage of the fact that equation (1.2) is basically coupled with the system (2.21) only through the source term $S$. This can be achieved by using separate (and thus, more accurate) estimates of the local speeds of propagation for the the transport equation (1.2) and the Saint-Venant system $(2.21)^{1}$. For equation (1.2), the local speeds are:

$$
\begin{aligned}
& a_{j+\frac{1}{2}, k}^{+}=\max \left\{u_{j, k}^{\mathrm{E}}, u_{j+1, k}^{\mathrm{W}}, 0\right\}, \quad a_{j+\frac{1}{2}, k}^{-}=\min \left\{u_{j, k}^{\mathrm{E}}, u_{j+1, k}^{\mathrm{W}}, 0\right\}, \\
& b_{j, k+\frac{1}{2}}^{+}=\max \left\{u_{j, k}^{\mathrm{N}}, u_{j, k+1}^{\mathrm{S}}, 0\right\}, \quad b_{j, k+\frac{1}{2}}^{-}=\min \left\{u_{j, k}^{\mathrm{N}}, u_{j, k+1}^{\mathrm{S}}, 0\right\},
\end{aligned}
$$

while for the Saint-Venant system (2.21) as well as for the extended system (2.21), (1.2) they are, in general, larger:

$$
\begin{aligned}
& a_{j+\frac{1}{2}, k}^{+}=\max \left\{(u+\sqrt{g h})_{j, k}^{\mathrm{E}},(u+\sqrt{g h})_{j+1, k}^{\mathrm{W}}, 0\right\}, \\
& a_{j+\frac{1}{2}, k}^{-}=\min \left\{(u-\sqrt{g h})_{j, k}^{\mathrm{E}},(u-\sqrt{g h})_{j+1, k}^{\mathrm{W}}, 0\right\}, \\
& b_{j, k+\frac{1}{2}}^{+}=\max \left\{(u+\sqrt{g h})_{j, k}^{\mathrm{N}},(u+\sqrt{g h})_{j, k+1}^{\mathrm{S}}, 0\right\}, \\
& b_{j, k+\frac{1}{2}}^{-}=\min \left\{(u-\sqrt{g h})_{j, k}^{\mathrm{N}},(u-\sqrt{g h})_{j, k+1}^{\mathrm{S}}, 0\right\},
\end{aligned}
$$

\footnotetext{
1 This idea has been worked out by A. Kurganov and G. Petrova, and was implemented in [5] in the 1-D case.
} 
where $u_{j, k}^{\mathrm{E}}=(h u)_{j, k}^{\mathrm{E}} / h_{j, k}^{\mathrm{E}}, h_{j, k}^{\mathrm{E}}=w_{j, k}^{\mathrm{E}}-B\left(x_{j+\frac{1}{2}}, y_{k}\right),(h u)_{j, k}^{\mathrm{E}}$ and $w_{j, k}^{\mathrm{E}}$ are obtained from the piecewise linear reconstruction (2.6), and the corresponding point values of $u^{\mathrm{W}(\mathrm{N}, \mathrm{S})}$ and $h^{\mathrm{W}(\mathrm{N}, \mathrm{S})}$ are computed similarly.

The numerical experiments in Section 3 and in [5] demonstrate that such a modification of the central-upwind scheme provides a relatively high resolution of the contact waves in pollution concentration. However, these waves are still smeared, and the proposed finite-volume-particle method clearly outperforms the finite-volume one.

\section{REFERENCES}

[1] E. Audusse, F. Bouchut, M.-O. Bristeau, R. Klein and B. Perthame, A fast and stable well-balanced scheme with hydrostatic reconstruction for shallow water flows. SIAM J. Sci. Comput. 25 (2004) 2050-2065.

[2] E. Audusse and M.-O. Bristeau, Transport of pollutant in shallow water. A two time steps kinetic method. ESAIM: M2AN 37 (2003) 389-416.

[3] D.S. Bale, R.J. LeVeque, S. Mitran and J.A. Rossmanith, A wave propagation method for conservation laws and balance laws with spatially varying flux functions. SIAM J. Sci. Comput. 24 (2002) 955-978.

[4] M.-O. Bristeau and B. Perthame, Transport of pollutant in shallow water using kinetic schemes. CEMRACS, Orsay (electronic), ESAIM Proc., Paris. Soc. Math. Appl. Indust. 10 (1999) 9-21.

[5] A. Chertock, A. Kurganov and G. Petrova, Finite-volume-particle methods for models of transport of pollutant in shallow water. J. Sci. Comput. (to appear).

[6] A. Cohen and B. Perthame, Optimal approximations of transport equations by particle and pseudoparticle methods. SIAM J. Math. Anal. 32 (2000) 616-636.

[7] B. Engquist, P. Lötstedt and B. Sjögreen, Nonlinear filters for efficient shock computation. Math. Comp. 52 (1989) 509-537.

[8] A.F. Filippov, Differential equations with discontinuous right-hand side. (Russian). Mat. Sb. (N.S.) 51 (1960) 99-128.

[9] A.F. Filippov, Differential equations with discontinuous right-hand side. AMS Transl. 42 (1964) 199-231.

[10] A.F. Filippov, Differential equations with discontinuous right-hand side, Translated from the Russian. Kluwer Academic Publishers Group, Dordrecht. Math. Appl. (Soviet Series) 18 (1988).

[11] T. Gallouët, J.-M. Hérard and N. Seguin, Some approximate Godunov schemes to compute shallow-water equations with topography. Comput. Fluids 32 (2003) 479-513.

[12] J.F. Gerbeau and B. Perthame, Derivation of viscous Saint-Venant system for laminar shallow water; numerical validation. Discrete Contin. Dyn. Syst. Ser. B 1 (2001) 89-102.

[13] S. Gottlieb, C.-W. Shu and E. Tadmor, High order time discretization methods with the strong stability property. SIAM Rev. 43 (2001) 89-112.

[14] A. Kurganov and D. Levy, Central-upwind schemes for the Saint-Venant system. ESAIM: M2AN 36 (2002) $397-425$.

[15] A. Kurganov and C.-T. Lin, On the reduction of numerical dissipation in central-upwind schemes (in preparation).

[16] A. Kurganov, S. Noelle and G. Petrova, Semi-discrete central-upwind schemes for hyperbolic conservation laws and Hamilton-Jacobi equations. SIAM J. Sci. Comput. 21 (2001) 707-740.

[17] A. Kurganov and G. Petrova, Central schemes and contact discontinuities. ESAIM: M2AN 34 (2000) 1259-1275.

[18] A. Kurganov and E. Tadmor, New high-resolution central schemes for nonlinear conservation laws and convection-diffusion equations. J. Comput. Phys. 160 (2000) 241-282.

[19] B. van Leer, Towards the ultimate conservative difference scheme, V. A second order sequel to Godunov's method. J. Comput. Phys. 32 (1979) 101-136.

[20] H. Nessyahu and E. Tadmor, Non-oscillatory central differencing for hyperbolic conservation laws. J. Comput. Phys. 87 (1990) 408-463.

[21] B. Perthame and C. Simeoni, A kinetic scheme for the Saint-Venant system with a source. Calcolo 38 (2001) 201-231.

[22] P.A. Raviart, An analysis of particle methods, in Numerical methods in fluid dynamics (Como, 1983). Lect. Notes Math. 1127 (1985) 243-324.

[23] A.J.C. de Saint-Venant, Théorie du mouvement non-permanent des eaux, avec application aux crues des rivières et à l'introduction des marées dans leur lit. C. R. Acad. Sci. Paris 73 (1871) 147-154.

[24] P.K. Sweby, High resolution schemes using flux limiters for hyperbolic conservation laws. SIAM J. Numer. Anal. 21 (1984) 995-1011. 